\title{
GLAD!
}

Revue sur le langage, le genre, les sexualités

$09 \mid 2020$

Traductologies féministes

\section{La violence par partenaire intime : enjeux traductologiques et politiques des catégorisations de la violence}

Intimate Partner Violence: Translation, Politics and Categorizations of Violence

\section{Samantha Saïdi}

\section{(2) OpenEdition}

Journals

Édition électronique

URL : http://journals.openedition.org/glad/2347

DOI : $10.4000 /$ glad. 2347

ISSN : 2551-0819

Éditeur

Association GSL

\section{Référence électronique}

Samantha Saïdi, "La violence par partenaire intime : enjeux traductologiques et politiques des catégorisations de la violence », GLAD! [En ligne], 09 | 2020, mis en ligne le 20 décembre 2020,

consulté le 20 janvier 2021. URL : http://journals.openedition.org/glad/2347 ; DOI : https://doi.org/ 10.4000 /glad.2347

Ce document a été généré automatiquement le 20 janvier 2021.

\section{c)}

La revue GLAD! est mise à disposition selon les termes de la Licence Creative Commons Attribution -

Pas d'Utilisation Commerciale - Pas de Modification 4.0 International. 


\title{
La violence par partenaire intime : enjeux traductologiques et politiques des catégorisations de la violence
}

Intimate Partner Violence: Translation, Politics and Categorizations of Violence

\author{
Samantha Saïdi
}

\section{Introduction}

1 Traduire des textes sur les violences dans les relations hétérosexuelles et homosexuelles amène à réfléchir aux rapports sociaux de sexes qu'elles manifestent, ainsi qu'aux enjeux de la circulation transnationale et transdisciplinaire de leurs analyses pour la lutte contre les violences faites aux femmes ${ }^{1}$. L'objectif principal de cet article est d'analyser des écrits qui ont combattu ces violences, comme ceux de Claire M. Renzetti et Michael P. Johnson, que j'ai été amenée à traduire ${ }^{2}$, ainsi que des enjeux interprétatifs, linguistiques et méthodologiques posés par leurs traductions et leurs réutilisations secondaires ${ }^{3}$, ceci afin d'élaborer les outils d'une pensée critique autour de l'idée d'une asymétrie genrée des violences par partenaires intimes (VPI) ${ }^{4}$.

2 Cette étude n'est pas une enquête de sociologie politique, mais une exploration de traductologie politique sur la circulation des concepts et les méthodes d'un champ d'études pluridisciplinaire, celui des violences par partenaires intimes (VPI). Aux ÉtatsUnis, les études sur Translation and Politics analysent le rôle de la traduction dans la transmission des idées politiques, les rapports des interprètes, traductrices et traducteurs avec le pouvoir, ainsi que les politiques de la traduction (Fernández \& Evans 2018). En France la traductologie a été repensée au prisme de l'idéologie (Guillaume 2016a, 2016b) ou du féminisme, comme nous le faisons dans le projet FELiCiTE Féminismes en ligne: Circulations, Traductions et Éditions (Saïdi, 
Mozziconacci 2018 ; Saïdi, Mozziconacci, Thomas \& Orazi 2018 ; Saïdi, Mozziconacci \& Orazi 2017) autour duquel nous avons coordonné ce numéro ${ }^{5}$.

3 Le champ d'étude anglophone Translation and Politics permet de délimiter le cadre d'un domaine de recherche que j'appellerai Traductologie Politique - plus précisément, Traductologie du fait social et politique pour expliciter la juxtaposition de traductologie et politique. Cette traductologie devrait permettre d'étudier sur le plan linguistique et méthodologique les traductions et les ré-usages transnationaux de textes et catégories produites en sciences humaines et sociales pour comprendre les relations de domination entre les personnes, les groupes humains ainsi que leurs rapports aux institutions et à l'État.

4 En traduisant l'article de Michael Johnson «Conflict and Control. Gender Symmetry and Asymmetry in Domestic Violence " (2006) et l'ouvrage de Claire Renzetti Violent Betrayal. Partner Abuse in Lesbian Relationships (1992), des questions récurrentes ont émergé qui débordaient le cadre des analyses féministes et ont été partagées à FELiCiTE (Saïdi 2019) et au laboratoire VisaGe (Saïdi 2020). Ces questions de traductologie politique seront développées ici. Elles interrogent la circulation des idées produites (typologies, concepts) pour analyser les violences conjugales, leur inscription dans les relations de domination entre les partenaires, et leur lien étroit avec les institutions et l'État. En effet, il s'agit de savoir si la circulation des écrits sur les VPI est empêchée ou désorientée par l'instabilité traductive de certains concepts clefs (terminologies et pluridisciplinarité), par la simplification des mésusages à visée patriarcale, ou par l'absence de traduction de centaines de textes du domaine. Des obstacles à la circulation viennent aussi de la polarisation politique ou disciplinaire des recherches. Cette polarisation est effacée en surface par des études qui évitent de s'appuyer sur les épistémologies du point de vue situé, ou de construire de nouveaux outils et de nouvelles méthodes scientifiques nécessaires à une compréhension transdisciplinaire des phénomènes sociaux. Je me concentrerai ici uniquement sur les deux textes de Johnson et Renzetti traduits en 2018 mais l'argumentation pourrait être étendue à la circulation des typologies et des idées produites par d'autres auteurices ayant travaillé sur les violences par partenaire intime.

5 Sur le plan de la linguistique, la traduction implique un rapport particulier entre la personne qui traduit et les énoncés portant sur les violences par partenaire intime : elle est et n'est pas l'énonciatrice initiale. Ce rapport dichotomique à la fois de rupture et d'identification (Culioli 1991) ${ }^{6}$ amène les questions suivantes. Comment résoudre le problème de l'ambiguïté ${ }^{7}$ sémantique et conceptuelle engendrée par la pluridisciplinarité du domaine des VPI et la carence de dictionnaires bilingues établis sur ce domaine? La définition des concepts et catégories est fondamentale à l'élaboration scientifique en sciences humaines et sociales. Mais elle est soumise aux ambiguïtés cognitives ${ }^{8}$ et disciplinaires. Comment dans ce cas prendre en charge un énoncé à la place de l'énonciateurice originale, sans résoudre ou expliciter cette ambiguïté inhérente au "manque d'harmonie préétablie» (Culioli 2000) entre les individus au plan cognitif et entre les disciplines? De plus, dans un cadre de recherche sur l'origine genrée des violences dans les couples hétérosexuels et homosexuels, quelles sont les représentations de sexe véhiculées par la langue source qui forme les textes ? Comment faire correspondre ces représentations, c'est-à-dire le signifié de la langue source, dans la langue cible sans lui «faire subir des distorsions étrangères et “contre nature" au niveau du signifiant»? (Chamberlain 1988, §15)? Comme le 
préconise Guillaume pour les textes véhiculant une idéologie (2016), mon hypothèse est que la circulation des études sur les VPI ne peut se faire, d'un point de vue scientifique, sans explicitation (reformulation ou apparat critique) des rapports entre recherche et terrain étudié, entre la recherche source et la recherche cible qui va utiliser ces études, entre terrain source et terrain cible, et entre la traductrice, l'éditrice et l'autrice originale. Sur le plan méthodologique, la traduction me permettra également de réfléchir à la réception de ces enquêtes dans l'espace francophone, mais aussi dans toutes les analyses secondaires (y compris anglophones) - considérées ici comme des formes de traductions.

Après avoir rappelé l'enjeu sociétal de ces questions pour la réception d'enquêtes étatsuniennes sur les VPI en France (1), j'exposerai les analyses morphosémantiques qui permettent de résoudre l'ambiguïté de genre ou directionnelle des violences dans les néologismes de catégorisation générique des violences (intimate partner violence, lesbian battering). L'enjeu sera d'éviter de suggérer dans les énoncés produits en français une bidirectionnalité ou essentialisation genrée des violences lorsqu'elles sont absentes en anglais (2). Je présenterai ensuite les questions d'interprétation sémantique et politique posées par la traduction de certaines catégories de violences, notamment par celle de la typologie de Johnson ou par celle de facteurs de violence étudiés par Renzetti pour comprendre les rapports entre les termes de violence et de control, terrorism, intimity, patriarchy, symetry et power (3). Les questions onomastiques posées par les patronymes et l'effacement des prénoms en bibliographie seront abordées au regard du genre (4). Enfin, j'expliciterai les carences méthodologiques des ré-usages (vus comme des traductions) de Johnson et Renzetti aux États-Unis et en France grâce à des exemples de réutilisations partielles ou réorientées et donc, pour certaines, de traductions fautives ou malhonnêtes (5).

\section{Contexte d'analyse : enjeu sociétal et approche traductologique}

\section{Enjeu sociétal du débat sur la bidirectionnalité des violences}

7 Dans Une ethnographie de la violence est-elle possible? Lenclud, Claverie et Jamin (1984) reviennent sur les difficultés et la légitimité des ethnographes à utiliser leur propre définition de la violence pour observer des faits violents, et les qualifier comme tels, sur un terrain empreint d'autres subjectivités sur les violences. Cette question est décuplée lorsqu'il s'agit de traduire les définitions produites dans un contexte culturel et social spécifique et une discipline précise, pour une réception transnationale. D'un point de vue sémantique, revenir sur la traduction de la typologie de Johnson et de l'appareil notionnel qu'il mobilise permettra de répondre aux questions de catégorisation des violences (Naepels, 2006), et de revisiter les enjeux politiques de l'interprétation des notions de terrorisme, intimité, patriarcat et symétrie en rapport avec celle de violence. D'un point de vue morphosémantique, revenir sur la traduction de matrices nominales par composition utilisées par Johnson ou Renzetti, comme intimate partner violence ou lesbian partner abuse qui ne portent ni marque du genre, ni préposition [article Ø] entre les noms pour spécifier un genre grammatical ou une direction de la violence, permettra de comprendre s'il existe, d'après les auteur.es étudiées, une origine genrée des violences, et de répondre à la question de la symétrie ou l'asymétrie des violences. 
Dans les années 1990 aux États-Unis un débat s'amorce autour de la question de la symétrie genrée des violences conjugales. Polariséees autour de cette question s'opposent les féministes et les spécialistes des études familiales. Le point de vue sociologique ou philosophique féministe dénonce le caractère patriarcal et structurel des violences (gender asymetry). Le point de vue sociologique et psychologique des approches familiales des violences (Kaufman, Harrison et Hyde 2014) s'appuie sur des sondages nationaux états-uniens de grande ampleur et donne lieu à des études représentant la violence comme un phénomène individuel ou induit par les interactions familiales, non-construit socialement, qui toucherait autant les femmes que les hommes (gender symetry), avec, par exemple, la figure du pervers narcissique ou le "Syndrome d'aliénation parentale » inventé par Robert Gardner et dénoncé par Léo Thiers-Vidal (2006). Du côté des études féministes, Michael Johnson propose une typologie de violences conjugales construite autour de la notion de contrôle. Il démontre comment les méthodes d'échantillonnage, et le fait d'ignorer sa typologie, et de ne pas mesurer le degré de contrôle des violences, peuvent biaiser les résultats des recherches et laisser croire qu'il existe une symétrie genrée dans les violences conjugales. Pour lui, il est surtout important de repérer si, à travers les violences produites, le bourreau exerce une volonté de contrôle total, par le recours à diverses stratégies d'emprise relevées par Ellen Pence \& Michael Paymar en 1993: menaces, contrôle financier, distribution de gratifications et de punitions, instrumentalisation des enfants, isolement, maltraitance émotionnelle active et passive, emprise sexuelle sans consentement ${ }^{9}$. Son travail a été repris dans une abondante littérature francophone en sociologie (Blondin, Ouellet, Leclerc 2018; Lemelin 2020). Sa typologie sera au cœur de notre analyse. J'étudierai également une autre enquête qui évalue le degré d'emprise exercé dans la violence sur partenaire intime : l'enquête participative que Claire Renzetti a élaborée en 1992 pour étudier les violences par partenaires intimes lesbiennes. Avant d'analyser les problèmes linguistiques posés par la traduction de ces deux enquêtes, voici quelques chiffres pour éclairer l'enjeu sociétal de la réception de textes étatsuniens sur les VPI en France. Les statistiques de différents ministères montrent que si les hommes subissent des violences, ils en sont les principaux acteurs, et les femmes, les principales victimes (cf. Tableau 1). Cette asymétrie statistique révèle des conditions de vie totalement asymétriques entre femmes et hommes en France. 
- Ministère de l'Intérieur : " En moyenne, chaque année entre 2008 \& 2016, un peu plus de 220000 personnes de 18 à 75 ans résidant en France métropolitaine ont déclaré avoir été victimes de violences sexuelles. Plus de $80 \%$ d'entre elles sont des femmes (184000 victimes par an en moyenne) ${ }^{10}$. »

- Ministère de la Justice : Dans Les condamnations pour violences sexuelles (2018), le ministère compte 68783 condamnations prononcées pour viol entre 2007 \& 2016 en France « dont $99 \%$ des condamnés sont des hommes ( $1 \%$ des Femmes) ${ }^{11}$ "

- Ministère de la Justice : Dans Les victimes du sexisme en France (2019), le ministère compte 17110 viols (ou tentatives) enregistrés en 2017 par les forces de sécurité, dont 14934 étaient des viols de femmes (12564 hors cadre conjugal et 2.370 par conjoints) ${ }^{12}$

- Haut Conseil de l'égalité, Amnesty International, ONVEF, Ministères français de la Justice et de l'Intérieur : ils rapportent que les femmes sont les principales victimes ${ }^{13}$ :

- D'attitudes ou décisions sexistes au travail ( $80 \%$ des femmes salariées) ;

- Du sexisme dont les plaintes sont enregistrées par les forces de l'ordre (89\% des femmes en 2016, avec sur 155398 victimes, 134850 femmes ; en 2017, 162940 victimes enregistrées, dont 141626 femmes) ;

- Des violences par partenaire : en 2017, 16829 par des hommes, contre 730 par des femmes ;

- Des violences par (ex-)partenaire : sur 219000 femmes majeures en 2017 : 51 pour viol (que des hommes condamnés) ; 1469 pour violences ayant entrainé plus de 8 jours d'ITT (1416 hommes condamnés ; 52 femmes condamnées) ; 1438 menaces/harcèlement (1413 hommes condamnés; 25 femmes condamnées); 205 agressions sexuelles hors viol (204 hommes condamnés et 1 femme) ; 42 non-respects d'une ordonnance de protection (uniquement des hommes condamnés) ;

- Des meurtres par un (ex-)conjoint. En 2017 : 130 femmes assassinées par (ex-)conjoint ; 21 hommes assassinés par (ex-)conjointe (dans $60 \%$ les femmes meurtrières avaient été victimes de violence par leur (ex-)conjoint) ${ }^{14}$. En 2018: 121 femmes assassinées par (ex-)conjoint ${ }^{15}$; En 2019 : 145 femmes assassinées par (ex-)conjoint ${ }^{16} ; 27$ hommes assassinés par (ex-)conjointe.

- Aucun ministère ne chiffre les meurtres de femmes transsexuelles comme celui de Vanessa Campos assassinée par une « brigade anti-trav ${ }^{17}$.

Figure 1 : Des agressions sexuelles aux féminicides : chiffres ministériels sur les violences hommes-femmes en France, pour comprendre le contexte de la réception des enquêtes sur les VPI.

\section{Approche linguistique et traductologique}

9 À partir d'exemples concrets de problèmes de traduction de catégories de violence et de leurs facteurs, il est possible de fonder une traductologie du fait social et politique, mais aussi de s'emparer d'un sujet de recherche en se posant la question de sa circulation pour en approfondir la compréhension, les concepts, et construire de nouveaux outils propres à une meilleure circulation transnationale. La traductologie a connu différents courants, comme la Théorie interprétative de la traduction (TIT) développée à l'ESIT (Seleskovitch \& Lederer 2009), la Théorie du skopos (Nord 1997) et la sémiotique. Sans nier l'apport des deux premières théories, j'aborde la traductologie par la linguistique interprétative, avec une approche inspirée de la Théorie des opérations énonciatives d'Antoine Culioli (TOE) - qui, si elle s'inscrit dans une démarche sémiotique, en bouleverse aussi les fondements et le formalisme. Tout d'abord, l'approche cognitive de la TIT est intéressante car elle distingue trois étapes pour la traduction: Compréhension de la langue 1/Déverbalisation/Reformulation dans la langue 2, mais elle ne fournit pas tous les outils qui auraient permis la représentation de 
cette déverbalisation hypothétique, ressentie empiriquement en contexte d'interprétariat simultané. La théorie du skopos ne me semble pas non plus pouvoir s'appliquer au corpus étudié puisque ce n'est pas l'utilité du texte cible qui est recherchée ici, mais la transposition et l'explicitation, dans le texte-cible, de catégories produites pour décrire un certain contexte-source fait de rapports de force. La TOE me permettait en revanche une approche linguistique polyvalente (à la fois sémantique, syntaxique et pragmatique) compatible avec une approche anthropologique ou sociologique. La TOE est à la fois une théorie de l'énonciation, une théorie du repérage notionnel et une théorie de l'invariance et de la variation dans les langues. Construite autour de l'étude, langue après langue, de notions et de leurs domaines notionnels, elle s'appuie sur les mécanismes énonciatifs et l'explicitation du contexte d'énonciation pour les étudier. En effet, pour Antoine Culioli (1990) ces mécanismes ne sont pas externes à la langue (contrairement à la TIT et la sémiotique de Pierce) mais sont inscrits dans le processus même de l'énonciation en contexte. Pour lui, les notions sont fluides et la bonne formation d'un énoncé ne réside pas dans sa justesse grammaticale, mais dans l'attestabilité du contexte d'énonciation qui entoure l'apparition d'une forme. Le formalisme qu'il met à disposition ne représente ni un métalangage, ni une méta-représentation des sujets parlants, mais une méta-représentation des opérations abstraites prédicatives.

Par ailleurs, Antoine Culioli, dont l'approche constructiviste se distingue pourtant de la linguistique cognitive - née vers 1956 et développée en parallèle aux États-Unis - a une approche intéressante de la cognition qu'il considère comme une notion « dangereusement ambiguë » $(1995$, p. 31) : pour lui, la cognition comprend toujours l'affect (1990: p. 21) et peut être représentée au premier niveau des trois niveaux de représentations qu'il dégage de l'énonciation (celui des représentations mentales ou cognitives, celui des représentations linguistiques ou textuelles et celui des représentations métalinguistiques) (Fuchs, 2008). Ici, je tenterai d'expliciter certaines catégories au niveau des "représentations mentales" (individuelles même si elles se font en interaction) et « textuelles ou linguistiques » (ici les catégories disciplinaires) grâce au niveau des « représentations métalinguistiques ». L'objectif n'est pas ici de comprendre ce qui se joue dans les VPI d'un point de vue cognitif ; cela pourrait être l'objet d'autres études.

11 Cette approche linguistique me semblait être la plus compatible avec le respect du contexte scientifique, social et politique de l'élaboration catégorielle nécessaire à la compréhension des VPI dans une situation source (ici les États-Unis pour simplifier), et avec leur transposition appropriée dans une situation de traduction (vers le français). Car si en TOE on analyse en général des énoncés produits à l'oral lors de conversations courantes ou préparées, comme les interviews, en tenant compte du contexte interactionnel (prosodie, gestuelle), il est possible d'interpréter l'énoncé scientifique écrit dans son contexte de production (terrain, débats scientifiques avec telle ou telle discipline). Par ailleurs la TOE permettrait de formaliser le rapport du/de la traducteurice à l'auteurice. Selon la $\mathrm{TOE}^{18}$, voici comment on formaliserait cette relation dichotomique : dans le contexte d'écriture original $\left(\mathrm{Sit} \mathrm{t}_{0}\right)$, l'énonciatrice $\left(\mathrm{S}_{0}\right.$, celle qui écrit que...) est aussi la locutrice (S1, celle qui dit/pense que...) : $S_{0}=\mathrm{S} 1$ (identification). Alors que dans le contexte de traduction ( $\left.S i t_{0}{ }^{\prime}\right)$, l'énonciatrice n'est plus la locutrice : $S_{0}$ ' $\neq$ de S1 (rupture) mais doit s'identifier à elle et dire Je quand l'autrice dit $I\left(S_{0}{ }^{\prime}=\mathrm{S} 1\right.$ ' = S1). 


\section{Analyses morphosémantiques : traduction, genre et ambiguïté directionnelle des énoncés sur les violences}

\section{Genre et généricité dans la catégorisation des violences}

12 Avant de me pencher sur les problèmes sémantiques posés par mon corpus de traduction, plus précisément par l'appareil notionnel mobilisé par Johnson et Renzetti pour répondre au débat sur la directionnalité des violences et pour comprendre les violences par partenaires intimes hétérosexuelles et homosexuelles, voici les analyses morphosémantiques (racine, composition, combinatoire, agencement des mots) de groupes nominaux génériques produits dans le domaine des VPI pour catégoriser les violences.

13 Lorsqu'on traduit des néologismes créés en anglais pour analyser les VPI (ex. intimate partner violence), le genre grammatical du français, aligné sur une bi-catégorisation des sexes (violence [du/de la/de?] partenaire intime), peut produire des contresens. Ce problème vient-il uniquement du fait que les genres grammaticaux ne sont pas alignés entre l'anglais et le français? Ou est-il renforcé par une directionnalité donnée à la matrice par une lexicogénèse qui permet la catégorisation de la violence autour d'une figure de victime? La directionnalité et la généricité de la matrice pourraient être mieux analysées, afin de résoudre la difficulté à choisir une préposition ( $\mathrm{du}, \mathrm{de}$ la, des, sur, par, etc.) dans les traductions en français.

\section{Intimate Partner Violence}

Tout d'abord, il est important de conserver en français la distinction typologique marquée par Intimate Partner Violence, au lieu de l'escamoter en traduisant par une catégorie plus connue en France comme violence de couple, violence domestique ou violence conjugale. Ces catégories existent déjà aux États-Unis sous les termes couple violence, domestic violence, et la distinction d'une catégorie construite autour de la figure de partenaire intime est le reflet d'un rapport de force dans la société entre différentes formes de défense des victimes de violence et les différentes formes de gouvernance. Or respecter au mieux la traductibilité du terme intimate partner violence est autant une façon de respecter la catégorie scientifique produite que de rendre compte de ce rapport de force. Ainsi, la première raison pour laquelle il ne semble pas pertinent de traduire le groupe nominal intimate partner violence par violence conjugale ou violence de couple (OMS), c'est que ces catégories donnent une image plus restrictive de la relation intime, alors que la catégorie intimate partner violence a été forgée aux États-Unis et au Canada afin d'être plus inclusive et représentative de toutes les formes de relation intime que le terme domestic violence. D'après Lessard \& al. elle prend en compte « la violence dans les relations amoureuses des adolescents et la violence post-séparation. » (2015: 2 note 1$)$.

Deuxièmement, les traductions d'intimate partner violences qui prédominent dans la littérature francophone - comme violence entre partenaires ou violence du partenaire (cf. 3 Bibliographie) - ne sont pas explicitées et restent problématiques car elles suggèrent soit le genre, soit la réciprocité des violences. Par exemple, traduire intimate partner violence par violence entre partenaires intimes avec la préposition entre et le pluriel - 
pourtant absents de l'original - n'est-ce pas déjà suggérer une réciprocité dans la violence conjugale? En effet, dans son Dictionnaire historique de la langue Française (2019), Alain Rey rappelle que la préposition entre a «pris une valeur nouvelle pour exprimer des rapports de réciprocité dans un ensemble ou parmi différents éléments, puis pour exprimer une comparaison (XVIe s.). [...ex.] (s'entre-tuer)». Or, la partie précédente montrait comment la symétrie dans les violences conjugales était non seulement remise en cause par Johnson, mais aussi problématique d'un point de vue notionnel. Par ailleurs, traduire intimate partner violence par violence du partenaire intime avec la préposition $d u$ (contraction de la préposition de et de l'article défini masculin le) suggère que l'agresseur est un homme ce qui, d'après Johnson lui-même, est majoritairement vrai dans les cas de terrorisme intime, mais pas dans les cas de violence de résistance, dont les femmes sont les principales actrices. Puisque l'objectif de Johnson est de savoir si les hommes et les femmes exercent les mêmes catégories de violences, il serait fâcheux d'ajouter une information fautive au nom du masculin universel.

Le danger interprétatif de transposer certains substantifs de genre neutre en langue anglaise nous amène à nous poser la question de l'origine de ce problème. Le français, resté binaire en genre, ne nous permet pas de traduire le substantif anglais partner (qui n'est ni féminin ni masculin) sans avoir à statuer sur le sexe des partenaires impliqués dans la violence. Mais le problème ne dépasse-t-il pas ici la question du genre ?

Si le problème était uniquement un problème qui concerne le genre grammatical, nous n'aurions pas rencontré de problème interprétatif avec les expressions comme lesbian abuse qui mettent en scène deux personnes du même sexe (cf. partie suivante). Voici différentes solutions pour rendre compte du genre neutre de l'anglais. On aurait pu utiliser l'écriture "non sexiste" (Chevalier, 2019) comme violence du/de la partenaire intime. On aurait aussi pu avoir recours à une transposition qui placerait la personne responsable de la violence au centre, avec l'écriture inclusive sur l'adjectif : partenaire violente, partenaire intime violent.e. Mais cette solution focalise sur une partenaire particuliere, ce qui ne convient pas. Le problème n'est donc pas qu'une question de genre grammatical. D'autres marqueurs qui ne relèvent pas uniquement du genre grammatical sont utilisés en anglais pour accentuer le niveau de généricisation du groupe nominal intimate partner violence et l'élever au rang de catégorie.

En linguistique, il existe plusieurs manières d'aborder ce groupe nominal. On peut l'appréhender en tant que néologisme ou que relation génitive. Si on s'appuie sur la modélisation des matrices lexicogéniques de Tournier $(1985,1991)$ pour comprendre le néologisme intimate partner violence, on a une matrice morphosémantique nouvelle, à la fois sur le plan du signifiant et du signifié, et composée par juxtaposition. On a trois formes de génitifs possibles, en 's, en of, ou avec l'article zéro (N’sN), (NofN) ou (NøN). Par une approche différentielle et énonciative, Petit (1991) étudie ces structures telles qu'elles se distinguent dans les énoncés mathématiques :

1. The Chaucy's theorem

2. Chaucy $\varnothing$ theorem

3. The theorem of Chaucy

19 Dans l'énoncé 2, l'utilisation du déterminant the n'est plus possible contrairement à l'énoncé 1 , car The ne viendrait pas déterminer le mathématicien Chaucy, mais le theorem qui est déjà déterminé par le génitif en 's. Pour ces deux constructions (en 's et avec l'article zéro Ø) se distinguent de celle en of qui fonctionne pour ajouter de l'information. Les structures en 's (N's N) et avec l'article zéro (Nom Ø Nom) sont les 
marqueurs d'une relation thématisée. Il fonde son analyse sur Adamczewski \& Delmas (1998) pour dire que «la structure spécifique NØN marque une notion complexe [...] une opération de complémentation sémantique (recharge sémantique) » (p. 98). C'est ce que nous observons avec intimate partner violence qui devient, grâce à la construction avec l'article zéro $\varnothing$ une notion complexe, c'est-à-dire une catégorie de violence à part entière dans le domaine des violences familiales ou interpersonnelles. Si on envisage les trois structures génitives potentielles de cette matrice morphosémantique on observe des différences de signification entre 1) The intimate's partner violence, 2) The violence of the partner et 3) Intimate partner violence) :

- The violence of (the)(her)(his) intimate partner.

- Intimate partner $\varnothing$ violence partenaire-là ou la violence de (son) (sa) partenaire intime (à lui/à elle), et parlent de la violence d'un ou d'une partenaire particulière. On a alors affaire un partenaire spécifique, un cas de violence précis. Ce n'est pas le cas avec la structure (3) qui utilise l'article zéro Ø.

21 Cette composition avec l'article zéro $\emptyset$ est un génitif à valeur générique, ou un génitif à valeur adjectivale générique (Bouscaren et Chuquet 2002), mais peut être aussi considérée comme la "marque d'une notion complexe ", explicitée par Adamczewski et Delmas (1998). On ne parle plus de la violence $d u$ ou de la, c'est-à-dire de la violence d'une personne spécifique, mais de la violence de toute's les partenaires qui sont violente.'s.

Plusieurs solutions existent en français pour rendre compte de ce sens. Même si cela semble contre-intuitif on aurait pu, comme le préconise Adamczewski \& Delmas cidessous, utiliser violence de partenaire intime avec le déterminant de quantité non genré (de). Cependant cette préposition (de) reste étrange et ambiguë ici (parle-t-on de la violence exercée par un bourreau ou de la violence subie par la victime ?). On aurait aussi pu avoir recours à une modulation "violence dans une relation intime » centrée autour de la relation, mais cette solution efface une catégorie de violence étudiée et construite par les spécialistes autour de la figure de l'agresseur (intimate partner), tandis que des typologies plus anciennes étaient construites autour de l'image de la victime (child abuse, women abuse). D'une part, cette distinction est essentielle et sera analysée dans la partie 3 qui proposera une typologie complémentaire. D'autre part, bien des violences dénoncées par la catégorie intimate partner violence n'ont pas lieu dans, mais au-dehors de la cellule du couple ou post-séparation.

Pour moins d'ambiguïté j'ai donc eu recours à une autre solution empruntée au domaine juridique. Cette solution consiste à adapter une expression du dictionnaire de Yang-Paya et Marcovici (2016) : « violence par conjoint, concubin ou partenaire lié par PACS » en l'étendant à tout type de rapports intimes (couples non mariés comme les couples d'adolescent·e's ou les couples séparés, les partenaires sexuels épisodiques, etc.), dans la traduction suivante : violence par partenaire intime. 


\section{Couples lesbiens et ambiguïté directionnelle des violences}

\section{Lesbian Battering \& Abuse : traduction et stéréotypes}

battering, est composée de deux noms, et non pas d'un adjectif suivi d'un nom. En effet, dans ces deux matrices qui représentent une catégorie de violence spécifique comprenant une catégorie de victime, le lexème lesbian ne correspond pas à l'adjectif lesbien(ne) mais au nom français une lesbienne. Cette composition par juxtaposition est construite sur le même modèle que child abuse/ child battering. De la même manière qu'on ne traduirait pas child abuse par violence infantile ou violence enfantine (mais par maltraitance envers les enfants) ou women abuse par violence féminine (mais par maltraitance envers les femmes, etc.), on sera donc attentive à ne pas la traduire par violence lesbienne, ou pire, par la violence lesbienne (Gabora, Stewart et Lilley 2005). Pour ne pas essentialiser les minorités lesbiennes comme violentes - ce qui favoriserait la diffusion d'un stéréotype, absent dans le texte original.

Étant donné que dans ce cas les deux membres du couple sont des femmes, on peut se poser la question suivante : en disant lesbian battering, Renzetti parle-t-elle de brutalité commise par une femme lesbienne (violence par une lesbienne), ou de brutalité subie par une femme lesbienne (violence sur une lesbienne) ? Selon Ingo Plag (2003) nous avons affaire ici à un nom composé endocentrique dont la tête sémantique est placée à la droite de la matrice (battering) et dont le nom antéposé vient spécifier la classe des violences pour en faire une catégorie (lesbian). Nous attribuons ces noms abuse et battering à une action (to abuse, to batter) menée sur la victime, présent dans le substantif antéposé dans l'expression (women, child, lesbian). Ces catégories sont traduites ainsi dans différentes sources (violences à enfants, violence sur enfant, voies de fait à l'encontre des enfants, etc.). 
Figure 2
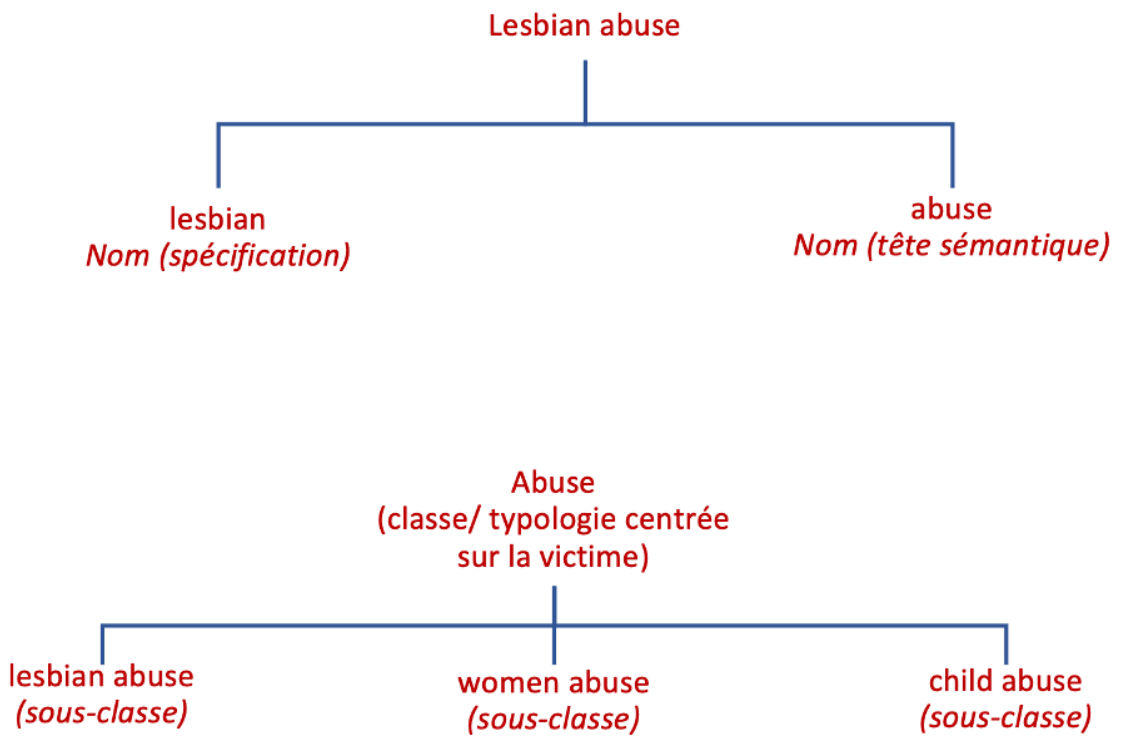

27 On traduira donc l'expression lesbian abuse par lesbienne maltraitée, ou par maltraitance envers une compagne lesbienne. De la même manière j'ai choisi de traduire lesbian battering par lesbienne battue ou lesbienne brutalisée.

\section{Lesbian Battering vs Intimate Partner Violence : la construction des classes}

On remarque que la spécification de la classe des violences est parfois inversée : tantôt on spécifie autour de la victime (lesbian abuse / women abuse), tantôt on spécifie autour de l'agresseur (intimate partner violence). 


\begin{tabular}{cccc}
\multicolumn{4}{c}{$\begin{array}{c}\text { Violence } \\
\text { (Classe/typologie } \\
\text { centrée sur } \\
\text { l'agressqur.euse) }\end{array}$} \\
$\begin{array}{c}\text { intimate partner } \\
\text { violence } \\
\text { (sous-classe } \\
\text { catégorie) }\end{array}$ & $\begin{array}{c}\text { men violence } \\
\text { (sous-classe } \\
\text { catégorie) }\end{array}$ & $\begin{array}{c}\text { women violence } \\
\text { (sous-classe } \\
\text { catégorie) }\end{array}$ & $\begin{array}{c}\text { couple violence } \\
\text { (sous-classe } \\
\text { catégorie) }\end{array}$ \\
& & &
\end{tabular}

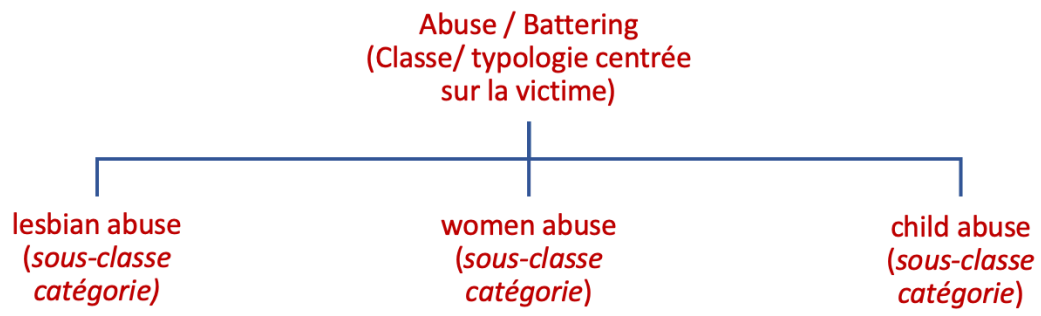

Comment une même construction morphosémantique (composition par juxtaposition avec l'article $\varnothing: N \varnothing N$ ) peut-elle produire ces inversions ? Comment un mot ou groupe de mot placé au même endroit dans la matrice peut être tantôt considéré comme un agresseur, tantôt comme une victime en anglais?

L'analyse des différents types de génitifs éclaire ces questions. Les génitifs en 's et en of sont possibles pour intimate partner violence, avec de petites distinctions de degré de généricité (cf. plus haut). Mais elles ne sont pas possibles pour lesbian abuse (abuse on a lesbian). La lexicogénèse parait différente pour les deux matrices: l'une semble être héritée de la forme génitive en $\varnothing$, l'autre semble être une juxtaposition avec une tête sémantique à droite (abuse ou battering) et un accusatif antéposé (women).

On remarque d'ailleurs que couple violence prend un nouvel éclairage interprétatif en français, puisque si la typologie en violence catégorise ses sous-classes comme des agresseurs, alors c'est bien le couple qui est considéré comme violent, ce qui suggèrerait une réciprocité des violences.

\section{Violent Betrayal : trahir sa classe de genre}

Dans l'ouvrage de Renzetti, on trouve d'autres difficultés de traduction dès le titre: Violent Betrayal. Partner Abuse in Lesbian Relationships. Tout d'abord, pour la première portion du titre, Violent Betrayal, j'ai eu recours à une transposition (Une violence déloyale) car un calque comme Trahison Violente équivalait ici à un contresens. En effet, la violence évoquée par Claire Renzetti dans le titre de son ouvrage n'est pas celle d'une infidélité qui serait vécue comme violente, mais bien celle d'actes de brutalités (verbales, psychologiques et physiques) qui, de son point de vue féministe, marquent une trahison à l'égard d'une solidarité (ou sororité) attendue entre femmes. Ensuite, pour la deuxième portion du titre, en disant partner abuse, parle-t-on de la maltraitance 
perpétrée, ou de la maltraitance subie ? Il semblerait que pour une anglophone c'est bien la victime qui est perçue dans cette expression. Quant à l'expression lesbian partner violence je l'ai traduite par violence par partenaire lesbienne comme nous l'avons vu plus haut avec intimate partner violence.

Nous reviendrons dans la partie suivante sur la construction des catégories autour des figures de la victime et de l'agresseur pour proposer une typologie complémentaire axée sur la projection sociale.

\section{Analyses sémantiques : catégorisation des violences et interprétation} question de la symétrie genrée des violences conjugales. Plutôt que de prendre part au débat, il fait un pas de côté pour expliquer que si les deux groupes parviennent à des données chiffrées justifiant deux discours opposés, c'est qu'il y a un problème dans la construction méthodologique de leurs recherches. Cette explication s'appuie sur une typologie des VPI en quatre catégories plus ou moins « enracinées dans un schéma de contrôle coercitif»: situational violence, intimate terrorism, violent resistance, mutual intimate terrorism (2010: 215). Grâce à cette typologie il effectue des réanalyses d'enquête (Frieze 1970) qui montrent : 1) que les chiffres des violences exercées par des hommes ou des femmes changent en fonction du type de violence ; 2) que les spécialistes des violences familiales et les féministes analysent des échantillons qui contiennent des catégories de violence différentes. La typologie vient avec des définitions affinées au fil de ses articles (Johnson 2006 jusqu'à 2017). La traduction des catégories et concepts de l'appareil notionnel de Johnson (2006) a fait surgir des questionnements sémantiques qui sont exposés ci-après.

\section{Control}

Comment traduire «control» en français? Par contrôle ou par emprise? Emprise semblait a priori plus approprié car ce mot sous-entend en psychanalyse cette pulsion de possession de l'autre qui est posé en objet qu'on souhaite s'approprier. Mais ce terme assignerait une tonalité plus psychanalytique à l'étude sociologique de Johnson. Ce problème de polysémie disciplinaire ou terminologique entre français courant et français psychanalytique - hérité de l'allemand - est posé en traductologie par Derrida dans L'oreille de l'autre: « [Q]uand, dans la métapsychologie, Freud parle de ceci ou de cela, des grands concepts analytiques, il ne les entend pas dans le sens courant de la langue ; Plaisir ne veut pas dire ce qu'on entend par plaisir ; Plaisir, c'est ce à partir de quoi le sens de plaisir peut se fixer. C'est-à-dire qu'il faut remonter (d'où le sens, d'où le mot de "anasémie") en deçà du sens pour comprendre comme le sens s'est formé ». Par ailleurs emprise escamoterait l'idée inhérente à control en sociologie, selon laquelle, dans les violences intimes, s'exerce également un rapport social de domination plus large qui relève du contrôle social des femmes (Hamner 1977 ; Guillaumin 1978). La solution adoptée est le terme "contrôle». Néanmoins, dans certains cas précis j'ai dû recourir à des explicitations dans les contextes où "contrôle " pouvait être confondu avec «maitrise » ou "emprise consentie ». Par exemple, dans le sous- 
titre, Sexual control pouvait laisser croire qu'il s'agissait soit d'une maîtrise contrôlée des rapports sexuels, soit d'une dépendance sexuelle d'une des deux partenaires alors que le paragraphe décrivait au contraire des rapports sexuels déplaisants, humiliants ou imposés comme une tactique de contrôle par partenaire. Il a fallu expliciter le soustitre grâce au contenu du paragraphe pour intituler la partie «Contrôle sexuel sans consentement ».

Une autre question pour les spécialistes des VPI, c'est de trouver de véritables outils pour mesurer le degré de contrôle exercé dans la VPI. Par exemple Jasinski, Blumenstein et Morgan (2014), ont démontré que c'est cette absence d'outils adéquats qui amène à faire perdurer un faux débat sur la symétrie des violences. Elles préconisent d'analyser finement dans quel contexte s'exerce ce contrôle. D'après leur exemple, un mari qui déclare que sa femme exerce un contrôle sur lui pourrait dire que sa femme tente de limiter ses contacts avec ses amis en omettant d'expliquer que c'est parce que ses amis ont des problèmes de toxicomanie, alors qu'une femme pourrait déclarer que son mari l'empêche d'avoir des contacts quand celui-ci le fait pour l'empêcher de se confier sur les maltraitances qu'elle subit. Pour Jasinski, Blumenstein et Morgan, l'outil de mesure du contrôle devrait couvrir un large panel de sujets comprenant, mais sans s'y limiter, le revenu familial, la situation professionnelle, les tactiques d'isolement, les menaces (crédibles ou non), la violence verbale et émotionnelle, et en particulier le niveau de crainte que chaque répondant ressent face à son conjoint. De ce point de vue elles estiment, comme nous le verrons en dernière partie, que l'apport de la typologie de Johnson est d'avoir permis de souligner cet aspect essentiel de la volonté de contrôle dans les VPI, mais que cette typologie doit être renforcée pour prouver une asymétrie ou symétrie des sexes dans les violences exercées.

\section{Typologie de Johnson}

\section{Situational Couple Violence}

Pour Johnson, ce type de violence, même s'il peut entraîner de graves conséquences, n'est pas dû à une volonté de contrôle, mais à un conflit ou un problème de communication qui dégénère. Il estime que si cette violence-là est symétrique au regard du genre elle reste potentiellement plus dangereuse pour les femmes que pour les hommes. En revanche elle n'est pas forcément amenée à se reproduire, ni à s'amplifier dans le temps. Après avoir hésité à utiliser «violences situationnelles ", ce qui a été le choix le plus courant dans la réception francophone canadienne du travail de Johnson (Lessard \& all 2015, Lapierre \& Côté 2014, 2018), j’ai finalement préféré l'expression de "violences circonstancielles de couple » " ou " contextuelles » pour éviter de suggérer une appartenance au situationnisme politique, linguistique, ou philosophique.

\section{Violent Resistance}

Pour Johnson la violent resistance renvoie à des actes de résistance utilisés par des personnes victimes de terrorisme intime. Il estime que cette forme de résistance, violente, est majoritairement employée par des femmes. Cette catégorie suggère a priori une violence «d'auto-défense». Cependant il semblait important de conserver 
l'idée de résilience et d'action également présente dans l'usage du substantif « résistance » qui évoque la force dans la durée. De la même manière que les violences de terrorisme intime sont évolutives et exponentielles dans le temps, on observe une résistance violente qui émerge avec le temps, avec des phases de lâcher-prise ou de stratégies d'acceptation (qui participent d'une résistance face aux violences) jusqu'au passage à l'action du refus de cette violence. Cette catégorie est très importante puisqu'elle redonne une agentivité aux victimes de violences (Marignier 2015).

\section{Intimate Terrorism}

39 La catégorie d'intimate terrorism : est un ensemble de tactiques d'emprise utilisées par une des deux membres du couple pour prendre le contrôle de tous les aspects de la vie de son/sa partenaire, l'assujettir, le/la maintenir au foyer et, pour cela, l'isoler, la/le surveiller, l'interroger, la/le dévaloriser, etc. Ces tactiques sont cycliques et évolutives dans le temps et ne peuvent que s'intensifier et s'aggraver, souvent même après une rupture du couple. Cette forme de violence est majoritairement employée par des hommes. Ces catégories de situational couple violence, violent resistance et intimate terrorism amènent à se pencher sur les rapports existants entre violence et les concepts de : terrorisme, intimité, patriarcat et (a)symétrie.

\section{Terrorism}

Du point de vue lexicographique la traduction de ce terme ne présente pas de difficulté (terrorisme). D'un point de vue sémantique et politique, la traductologie nous enjoint à nous poser la question de la légitimité du concept à traduire. Cet usage inédit de "terrorisme" ou "terroriste" chez Johnson pour qualifier une relation intime (intimate terrorism) permet de mettre le doigt sur le déséquilibre existant entre les politiques publiques mises en place pour lutter contre les terroristes publics et les terroristes intimes. Malgré le nombre de féminicides important en France (Tableau 1), les moyens restent insuffisants. De plus, le nombre de femmes victimes de féminicides perpétrés par des partenaires transphobes ou des brigades anti-trav' ne sont pas officiellement comptabilisés.

41 La catégorie d'intimate terrorism est originale et préfigurait d'autres travaux qui ont, par la suite, élaboré leur théorie autour de l'idée que la violence par partenaire intime est une atteinte aux droits humains et un fléau social. Evan Stark par exemple, sociologue féministe expert du système judiciaire et des violences conjugales, assimile les maltraitances ancrées dans le contrôle coercitif à des crimes contre les droits humains des femmes et «d'atteinte à la liberté » comme "[1]es prises d'otages, [1]es enlèvements, [1]es actes de torture et [1]es autres crimes de "séquestration" » (2012:8) dont les effets à long terme sur la victime sont dévastateurs. Il met en parallèle les liens entre les pouvoirs coercitifs domestiques et institutionnels sur les femmes. Cette vision fait écho aux analyses de Hanmer 1977, Guillaumin 1978 et Wittig 1982. Elle s'appuie sur celle de Robert J. Lifton $(1974,2017)$ ou de Lenore Walker (1989), spécialiste du syndrome de stress post-traumatique des victimes (2007). Ainsi, Stark critique les procédures et les institutions qui empêchent une prise en charge efficace des VPI. Pour lui, ce contrôle coercitif domestique, exercé dans un contexte social déjà inégal et contrôlant pour les femmes, s'apparente à celui qu'on fait subir aux adeptes de sectes, aux militaires et aux prisonniers de guerre : 
[L]es femmes peuvent contrôler autant que les hommes. Cependant, lorsque le délinquant est un homme, le contrôle coercitif exploite et renforce les inégalités sexuelles de l'ensemble de la société d'une manière qui la rend beaucoup plus dévastatrice que lorsque les femmes exercent un contrôle. $(2012 a: 5)^{19}$.

le contrôle coercitif est "genré » parce qu'il est utilisé pour garantir le privilège masculin et que son régime de domination/subordination est construit autour de l'application de stéréotypes sexistes. La "domination" désigne ici à la fois le pouvoir/privilège que le contrôle coercitif permet d'exercer dans les relations individuelles et le pouvoir politique qu'il offre lorsque les hommes, en tant que groupe, utilisent leurs tactiques oppressives pour renforcer les inégalités sexuelles persistantes dans la société en général. (2012a, p. 8)

\section{Intimity}

On aimerait explorer la notion d'intimité présente dans la catégorie intimate terrorism, mais aussi, de manière plus générale, dans intimate partner violence ${ }^{20}$. En effet, dans les articles étudiés le/la "partenaire intime " est toujours envisagée comme membre d'une dyade ou d'un couple: un compagnon ou une compagne, mais rarement une partenaire sexuellle. En effet, l'adjectif intime est souvent utilisé pour parler d'un lien étroit entre deux personnes, mais il qualifie également ce qui relève du domaine du privé, ce qui est caché, ou des relations charnelles. Par conséquent, comme annoncé par Lessard \& al. (2015), la catégorie de violence par partenaire intime devrait inclure les violences exercées par des partenaires sexuels de cadres moins formels que ceux du couple ou de la conjugalité, comme ceux des milieux festifs ou prostitutionnels. Dans Sociologie de la prostitution (2015), Lilian Mathieu évoque les violences exercées dans le milieu de la prostitution et nous aide à mieux comprendre le continuum des violences (Kelly 1987) exercées intimement (logiques internes), et socialement (logiques externes), envers les femmes qui illustrent le continuum des violences.

On trouve ces logiques internes dans les violences de type terrorisme intime exercées dans le couple (affirmer son pouvoir, contrôler son partenaire, s'octroyer son argent, sauvegarder sa dignité de partenaire). Les logiques externes sont présentes dans tous les espaces extra conjugaux et permettent d'asseoir le pouvoir institutionnel, de contrôler le rôle des femmes dans la société, de s'octroyer le fruit du travail des femmes, et de préserver la dignité patriarcale dans la société et les institutions (ex. Académie Française).

\section{Patriarchy}

Johnson utilisait la catégorie de patriarcal terrorism (1995) avant de la remplacer par intimate terrorism pour la raison suivante : 
I still believe that most intimate terrorism is perpetrated by men in Je crois toujours que la plupart du terrorisme intime est heterosexual relationships and that in such cases the violence is indeed rooted in patriarchal traditions. However, it is clear that there are women intimate terrorists in heterosexual and same-sex relationships (for descriptions of intimate terrorism in lesbian relationships, see Renzetti, 1992). Furthermore, it is not clear that all intimate terrorism, even men's, is rooted in patriarchal ideas or structures. perpétré par des hommes dans le cadre de relations patriarcales et que, dans ces cas-là, la violence est en effet enracinée dans les traditions patriarcales. Cependant, ce qui est clair c'est qu'il existe des femmes qui sont des terroristes intimes dans les relations hétérosexuelles et homosexuelles (pour les descriptions de terrorisme intime dans les relations lesbiennes, voir Renzetti, 1992). Ce qui n'est pas clair en revanche, c'est que tous les cas de terrorisme intime, même ceux perpétrés par des hommes, soient enracinés dans les idées ou structures patriarcales. " (Johnson $2006: 2^{21}$ )

\section{Ne pas confondre les visées individuelles et les visées structurelles}

Même s'il rejette finalement cette catégorie de patriarcal terrorism et le fait qu'elle puisse expliquer tous les types de violence, il nous semble intéressant de réfléchir à son apport pour la compréhension des VPI. En effet, la catégorie de violence du terrorisme patriarcal est intéressante. Même si, comme Johnson, je ne pense pas que la violence de terrorisme intime soit uniquement "enracinée dans les idées ou structures patriarcales ", mais qu'elle relève souvent de la psychiatrie, elle ne peut pour autant échapper aux structures patriarcales qui viennent l'encourager et en renforcer la portée. Une violence dite " patriarcale ", ne doit pas être réduite à la violence du père, des hommes ou à la violence masculine. Elle n'est quasiment jamais exercée en conscience avec une visée patriarcale. Mais le résultat n'en est pas moins là : le maintien des femmes dans un certain ordre social. Il est donc plutôt question ici d'une violence qui, quelle que soit son origine (biologique, sociologique, physiologique, psychologique), s'exerce au profit d'un système patriarcal et au détriment des femmes comme groupe social de sexe. Émerge alors un biais dans les catégorisations des violences: les catégorisations de violences se sont jusqu'ici soit concentrées sur les victimes (child, women, lesbian violence), soit sur l'agresseur (intimate partner violence) et, grâce à Johnson sur la visée ou le degré de contrôle que cet agresseur exerce dans la violence. Cependant cette vision n'est pas complète, et devrait être approfondie par une typologie centrée autour des effets produits dans la société, comme nous le montrerons plus bas (cf. partie Pour une typologie complémentaire axée sur la projection sociale). Cela permettrait de résoudre des problèmes méthodologiques et de mésusage des typologies de Johnson par les masculinistes (Lapierre \& Côté 2018 ; Déri, Bard et Blais 2019).

\section{Humiliation du pouvoir et violences punitives}

Mais qu'est-ce que ce système défend au juste ? À partir de la définition que Christine Delphy développe dans L'Ennemi principal ${ }^{22}$, le patriarcat peut être vu comme un système social où les relations économiques, politiques, intellectuelles et émotionnelles sont édictées par des lois qui subordonnent les femmes à des rôles (gratuits ou salariés) sexuels, reproductifs, sociaux, créatifs ou de travail qui bénéficient à une société dominée et régie majoritairement par des hommes et les maintiennent au pouvoir. Ces rôles ont une valeur marchande et intellectuelle qui est souvent niée ou sous-évaluée 
pécuniairement et symboliquement (Marry \& Bereni 2017). Les femmes peuvent certes, de plus en plus, y déroger, avec combativité (vie autarcique communautaire, célibat, autonomie financière, etc.). Mais si le patriarcat est aussi une «dignité de patriarche (1594)» (p. 2598) comme nous le rappelle Alain Rey dans son Dictionnaire étymologique de la langue française (2019), ces femmes prennent le risque d'être soumises à des violences punitives de tout ce qui vient humilier cette dignité. Ces violences sont : symboliques (exclusion, abandon, isolement), physiques (coups portés), sexuelles (agression, harcèlement sexuel, viols) ou fatales (féminicides).

47 Ainsi, déroger au silence attendu par la classe dominante expose les femmes à des violences punitives symboliques, comme l'exclusion. Cette violence punitive symbolique qui défend la dignité du patriarcat s'est exprimée récemment lors de la distribution des Césars de la honte ${ }^{23}$ en février 2020 qui a récompensé Polanski (reconnu coupable aux États-Unis de viol sur mineure) et corrigé l'actrice Adèle Haenel qui venait de dénoncer le harcèlement sexuel que lui avait fait subir, lorsqu'elle était mineure, le réalisateur Christophe Ruggia ${ }^{24}$.

Déroger à une apparence de genre attendue et trahir la classe de sexe qu'on nous a attribué à la naissance (Beaubatie 2019) soumet également les hommes et les femmes à des violences punitives. Si une femme trahit la classe de sexe (homme) qui lui a été imposée à la naissance, elle s'expose à des violences qui peuvent aller jusqu'au meurtre. Rappelons les brigades anti-trav' évoquées plus haut qui ont assassiné Vanessa Campos en 2018. Ces violences meurtrières restent sous-évaluées malgré les appels d'associations de défense des droits des personnes transsexuelles comme Chrysalide.

À partir de ces définitions, il me semble que les typologies des VPI devraient mieux prendre en compte le contexte social dans lequel s'exerce la violence comme nous allons le voir ci-dessous.

\section{Pour des typologies précisant le groupe social de la victime}

50 Les violences de contrôle ne concernent pas seulement l'espace privé et intime, c'est pourquoi la catégorie violence par partenaire intime continue finalement d'individualiser le problème de la violence qui est un continuum depuis l'espace public jusqu'à l'espace privé. Il serait intéressant de recourir à des typologies qui prendraient mieux en compte l'influence du groupe social de la victime sur l'exercice des violences. Cette manière de procéder trans-typologique permettrait d'éviter les amalgames et d'avoir une approche plus respectueuse des approches intersectionnelles des VPI. Par exemple, si Straus et Gelles dénonçaient en 1975 des institutions qui avaient fait exploser le nombre d'afro-américains emprisonnés pour violence conjugale, Stark expliquait également (2007) que les femmes afro-américaines, souvent travailleuses et pourvoyeuses financières du foyer, avaient une habitude d'indépendance économique plus forte que les femmes blanches vis-à-vis de leur compagnon, ce qui leur faisait avoir recours plus facilement à la police et aux refuges.

51 Une prise en compte du contexte social d'exercice de la violence semble d'autant plus nécessaire que Renzetti remarque dans son étude sur les couples lesbiens qu'à l'intérieur du couple, les rapports peuvent parfois s'inverser entre groupe social dominant à l'extérieur et groupe social dominé. Dans son enquête, les personnes qui disposaient d'un statut social plus important ou plus prestigieux à l'extérieur, étaient parfois victimes de violences au sein du couple. Dans ce cas la violence subie à 
l'extérieur était reportée à l'intérieur par le partenaire violent sur le partenaire socialement dominant.

Une explicitation du contexte permet également de sortir des préjugés selon lesquels une violence commise par une femme lesbienne ne serait pas une violence à visée patriarcale, reproduisant celle du cadre hétéronormatif ${ }^{25}$ (Watermez 2012), tout en continuant à utiliser une typologie fondée sur l'objectif de l'agresseur comme celle de Johnson. Il ne s'agit pas ici de dire que si tel groupe social subit des discriminations, alors la violence s'exercerait systématiquement à l'encontre d'une de ses membres. Il s'agit plutôt d'étudier, dans un cas de VPI avéré, comment cette violence particulière s'appuie sur les violences ou discriminations envers le groupe auquel la victime appartient.

Par exemple Jasinski, Blumenstein et Morgan (2014) ou Nybergh, Enander, Krantz (2016) qui ont utilisé la typologie de Johnson pour étudier des cas d'hommes victimes de VPI, ont montré que ces hommes ne souffrent pas des mêmes conséquences que les femmes. Contrairement aux femmes, ils ne sont pas touchés, ou moins, par le PTSD, les arrêts de travail, les dépressions, etc. Dans leur cas, il semble que la violence ne soit pas aggravée par le contexte social dans lequel ils évoluent.

\section{(A)symetry}

54 Il est à noter que l'adjectif symétrique est utilisé dès 1977 par Jalna Hanmer pour dénoncer le rôle de l'État dans le renfort des rapports de subordination asymétrique des femmes aux hommes et d'une dépendance symétrique : " [Nous] analyserons le rôle de l'État dans la création d'une dépendance "symétrique" entre les sexes ». Comme nous l'avons vu plus haut, ce terme est au centre du débat qui a eu lieu dans les années 1990/2000 aux États-Unis autour de la question de la symétrie/l'asymétrie des violences conjugales au regard du genre (« Gender Symmetry and Asymmetry » Johnson 2006).

Rappelons que symétrie est catégorisé, en linguistique morphosémantique, comme une métaphore. Cette métaphore de la symétrie des violences renferme un impensé intrinsèque qui aurait dû révéler la prévalence de situations de violences inversées (violence d'attaque vs violence d'autodéfense). En effet voici une image de forme symétrique par réflexion :

Figure 4 : Ségrégation horizontale et ségrégation verticale

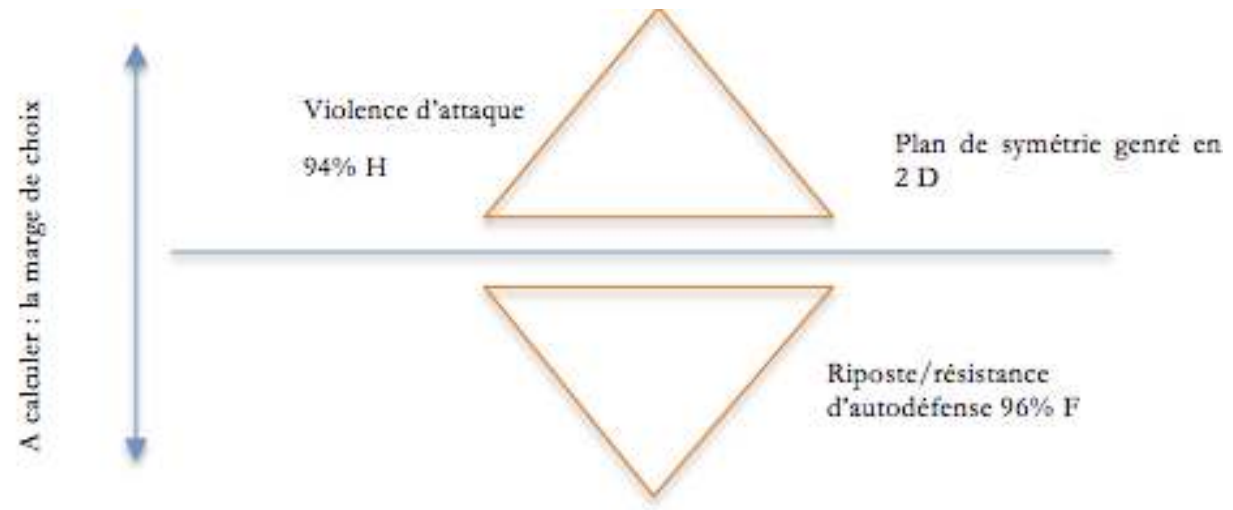

Cette image représente une symétrie en miroir (il en existe d'autres comme la symétrie par rotation, symétrie par translation, etc.). Tous les points reportés sont inversés à 
partir de la ligne médiane qui coupe une ligne de gradation qui pourrait représenter une marge de choix (choix d'attaquer/choix de riposter). C'est une transformation géométrique du plan qui modélise un pliage ou un effet miroir : ci-dessus, je l'ai dessinée d'après un reflet horizontal. Or, le fait que la métaphore mathématique de la symétrie des violences de couple ait été utilisée si longtemps par les spécialistes des violences familiales pour parler de bidirectionnalité des violences est symptomatique d'un refoulement de la valence différentielle des sexes (Héritier 1996) qui pénalise les femmes non seulement de manière horizontale (ségrégation horizontale avec des fonctions différenciées pour les hommes et les femmes) mais aussi verticale (ségrégation verticale avec des fonctions hiérarchisées entre hommes et femmes, et des positions subalternes pour les femmes).

57 Il semble que, dans l'imaginaire des spécialistes des violences familiales, la symétrie genrée ait été vue comme complémentaire, avec des fonctions divisées (et des coups portés) de manière horizontale : une symétrie horizontale des violences de part et d'autre d'un axe vertical (fig.2).

Figure 5 : Axe de symétrie vertical - Symétrie horizontale des violences

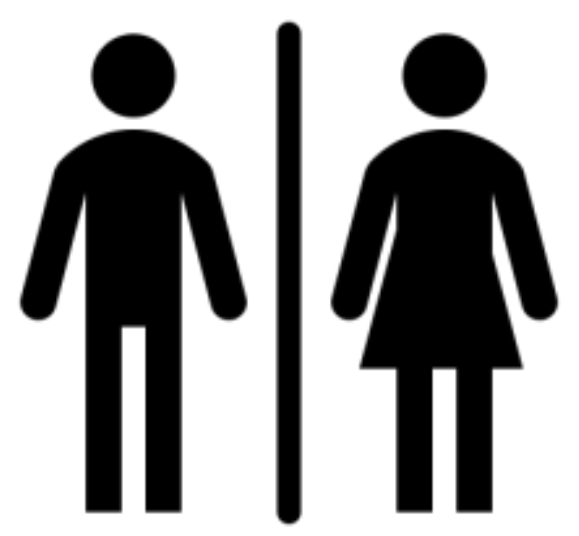

Mais avec un axe horizontal, on découvre une symétrie verticale des violences. Et dans ce cas-là, lequel des deux partenaires se trouve du bon côté du plan de symétrie ? L'homme ou la femme? Cette symétrie autour d'un axe horizontal fait écho à la ségrégation verticale qui introduit la hiérarchie en faveur l'homme sur la femme (fig. 3) : 
Figure 6 : Axe de symétrie horizontal - Symétrie verticale des violences

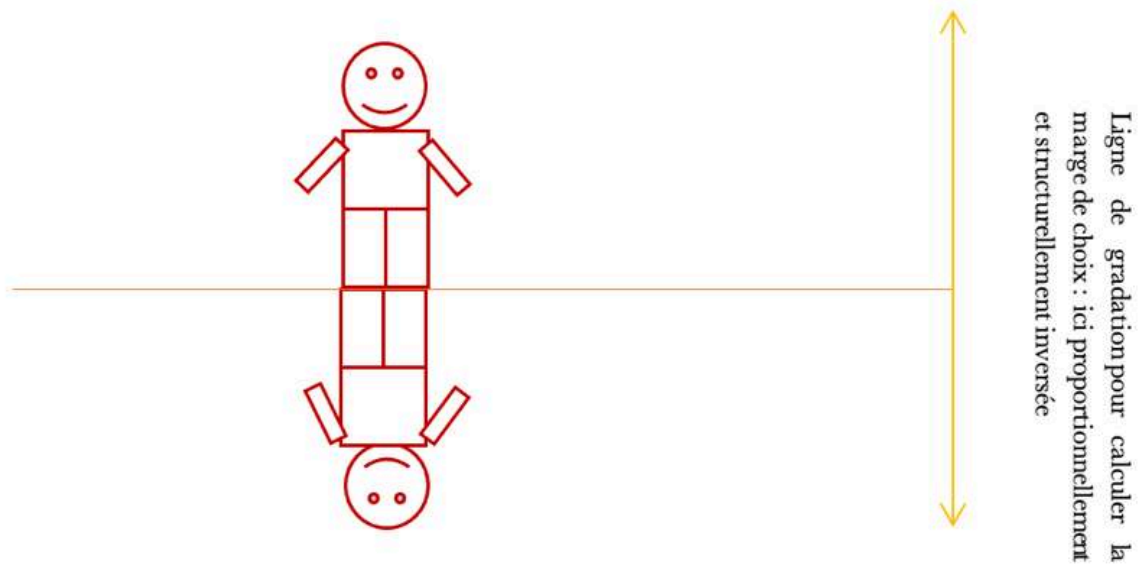

Dans sa réanalyse d'une étude menée par Frieze à Pittsburg en 1970, Johnson (2010) révèle justement que, parmi les enquêté'e's, les terroristes intimes sont des hommes à 96,9\% (94 sur 97 cas de terrorisme intime) et que $96 \%$ des personnes utilisant la violence de résistance sont des femmes (dans 53 cas sur 77 il s'agit d'auto-défense). La marge de choix dans l'utilisation de la violence est donc inversée pour l'homme et pour la femme (décision d'agression vs nécessité de se défendre). L'origine de ce type de violences n'est, pour l'homme et la femme, ni identique, ni mutuelle, ni simultanée : l'une déclenche l'autre. Johnson réfute l'idée d'une réciprocité genrée des violences dans le couple pour les cas de terrorisme intime et d'auto-défense, même si elles sont corrélées.

Il semblerait opportun d'abandonner la métaphore de la symétrie sexuée des violences, ainsi que les recherches statistiques sur la symétrie sexuée des violences afin de privilégier des recherches autour du degré de contrôle qui accompagne les VPI, et de comprendre comment s'organise la bidirectionnalité des violences. En effet, symétrie des chiffres (qui n'existe pas en France comme le montre notre tableau fig. 1) ne veut pas dire égalité de traitement et des conséquences.

\section{Analyse onomastique : patronymes, prénoms et genre}

61 Je me suis référée aux critiques féministes faites à l'invisibilisation des femmes (Chamberlain 1988) pour traduire la bibliographie de l'ouvrage de Claire Renzetti, dans « une langue non sexiste » (Chevalier 2019), c'est-à-dire dans une forme qui redonne de la visibilité aux femmes grâce à l'ajout de leur prénom. En effet, en parcourant la bibliographie dédiée aux violences dans les couples lesbiens ma première réaction fut d'être surprise qu'autant d'hommes aient travaillé (depuis les années 70), sur ce sujet d'apparence mineure. Puis, j'ai réalisé qu'il s'agissait d'un biais de lecture de ma part : j'avais figuré des hommes derrière les noms de famille Kaufman, Walker, Harrison, etc. alors qu'il s'agissait de près de cent-vingt femmes qui avaient travaillé à l'écriture des cent-trente études de violences de couple utilisées par Claire Renzetti: Phyllis A. Kaufman, Lydia Walker, Elizabeth Harrison, etc. 
62 La place prépondérante et de domination des hommes à l'université dans les décennies passées (Rogers \& Molinier 2016) peut renforcer ce biais de représentation, qui peut être plus ou moins prégnant selon l'âge ou les disciplines où l'on exerce. Il me semblait donc important de lever l'ambiguïté quant au genre des auteurices de la bibliographie. L'écriture non sexiste permet de féminiser lorsqu'on parle des femmes, de masculiniser lorsqu'il s'agit d'hommes, ou de marquer le masculin et le féminin lorsque la situation est ambiguë. Dans le cas d'une bibliographie les prénoms servent cette désambiguïsation, et une recherche sur ces prénoms m'a permis de compléter chaque référence bibliographique de l'ouvrage de Renzetti.

Comment expliquer, linguistiquement, que les noms propres limités au nom de famille puissent suggérer la masculinité ? Si on effectue une recherche sur le genre des noms suscités, certains ont un genre grammatical masculin avéré comme Walker, Harrison. D'autres ont le terme homme (man) dans leur composition (Kaufman, Coleman). Paul Siblot (1997) considère que nommer « est aussi l'exercice d'un pouvoir qui en modifiant le statut de l'être nommé change ce dernier lui-même. Nommer, c'est classer dans une catégorie linguistique en même temps qu'assigner une place dans l'ordre du monde » (1997 : 42).

64 Si nommer c'est classer, il est logique que les noms de famille puissent désigner des hommes dans l'inconscient collectif. En effet, d'une part le nom de famille est encore, en français et en anglais du moins, le symbole de la filiation patrilinéaire et du père de famille qui a le pouvoir de donner son nom à femme et enfants (patronyme). D'autre part, comme nous l'avons dit plus haut, dans le cas d'une bibliographie universitaire ce biais peut être amplifié par un contexte qui favorise la présence et la reconnaissance des hommes (Marry et Bereni. 2017).

Il est à rappeler que dans certaines langues les noms de famille sont féminisés lorsqu'ils désignent des femmes. Par exemple en russe, on dira Madame Berberova et Monsieur Berberov (Masculin :-OB (-ov); -EB (-ev)//Féminin:- -OBA (-ova); -EBA (-eva), ce qui n'est le cas, ni en anglais, ni en français. En revanche on aura besoin, en anglais comme en français du prénom pour redonner de la visibilité aux femmes qui ont travaillé sur les violences conjugales.

\section{Méthodologie : ré-usage comme une traduction}

Les réutilisations (reuse en anglais) et réanalyses (Duchesne, 2017) - que ce soit sous forme de synthèse, note, critique, analyse secondaire, etc. - seront considérées dans ici comme des véhicules de circulation des analyses sur les VPI, et seront donc envisagées comme des formes de traduction. En m'appuyant, entre autres, sur Johnson (2006), Small (2009) et Sophie Duchesne (2017) qui ont débusqué les manquements ou incompatibilités méthodologiques de certaines enquêtes ou de leur réanalyse, il semble nécessaire de clarifier les objectifs et méthodologies employés dans les enquêtes sur les VPI (Cavalin 2013) et aussi dans leurs réutilisations en contexte transnational. Par exemple si l'objectif principal de Johnson était de comprendre la nature des VPI en en définissant une typologie à partir d'une analyse secondaire de données récoltées par une recherche précédente, celui de Renzetti était de savoir selon quels motifs et mécanismes les violences par partenaire s'exerçaient envers les partenaires qui 
déclaraient des violences à travers la construction participative (avec des lesbiennes battues) d'une enquête.

Or, dans la réception ou la réutilisation du travail de Johnson, le contexte, la langue, et ces objectifs peuvent différer ${ }^{26}$. La réception de la typologie de Johnson, abondante (Blondin, Ouellet et Leclerc 2018 ; Dumont 2017), représente des formes de traductions partielles, que j'appellerai traductions secondaires, aux objectifs protéiformes. Certaines spécialistes s'emparent de cette typologie pour en vérifier la solidité et analyser leurs résultats d'enquêtes, comme Jasinski, Blumenstein et Morgan (2014) ou Nybergh, Enander, Krantz (2016) l'ont fait pour analyser les violences subies par les hommes. D'autres spécialistes, ont utilisé cette typologie pour défendre des propos contraires à ceux de Johnson, et en faveur d'une vision bidirectionnelle (Archer 2000) ou antiféministe (Lapierre \& Côté 2014, 2018) des violences familiales: il s'agit là de détournements. D'autres spécialistes, enfin, expriment une opposition féministe marquée à Johnson mais argumentent leur opposition, sans nier ses propositions de départ.

\section{Méthodologie d'échantillonnage et symétrie}

Comme nous l'avons dit en partie 2, Johnson apporte une critique méthodologique constructive au débat polarisé autour de la question de la symétrie des violences au regard du genre. Il relève le manque d'homogénéité, ou de comparabilité entre les données des grands sondages états-uniens effectués auprès de la population générale (utilisés par les spécialistes des violences familiales) et les données des études féministes issues d'hébergements d'urgence, de tribunaux. Il démontre aussi comment les méthodes d'échantillonnage, le fait d'ignorer qu'il existe différents motifs de VPI, et celui de ne pas mesurer le degré de contrôle accompagnant les violences, peuvent biaiser les résultats des recherches et laisser croire qu'il existe une symétrie genrée dans les violences conjugales. Johnson dénonce ainsi la prétendue impartialité ou représentativité des grands sondages utilisés par les spécialistes de la violence familiale :

Figure 7 : (Johnson, $2006: 1004^{27}$ ).

\begin{tabular}{ll|l} 
The final samples of so-called random & Bien entendu, si on prend en considération les taux de refus \\
sample surveys are, of course, not & de participer à l'enquête, les échantillons finaux de sondages \\
random, due to refusals. I estimated, for & dits aléatoires ne sont pas aléatoires. J'ai par exemple estimé \\
example, that the refusal rate in the & que pour les sondages nationaux sur les violences familiales \\
National Family Violence Surveys was & (National Family Violence Surveys) les taux de refus de \\
approximately 40\% rather than the 18\% & participer étaient approximativement de $40 \%$ au lieu des \\
usually reported (Johnson, 1995). & $18 \%$ rapportés habituellement (Johnson 1995).
\end{tabular}

69 Voyons maintenant des exemples de réusages de son travail, et ce que cela apporte d'un point de vue méthodologique à l'étude de Johnson et aux spécialistes de VPI en général. Je précise qu'une étude plus systématique de la réception de la typologie de Johnson pourrait être entreprise par les sociologues, car ici je ne donne que les quelques exemples que j'ai utilisés en tant que traductrice. 


\section{Réception des spécialistes des violences familiales}

70 Du côté des spécialistes des études familiales de la violence, comme le rappelle en 2006 Johnson lui-même, aucun de ses détracteurs de l'époque ne s'était véritablement emparé de cet apport méthodologique qui aurait mérité d'être ré-utilisé dans leurs enquêtes. Il parle ici de la réception de son travail chez Archer (2000) :

Figure 8 : Johnson $(2006: 1004)^{28}$.

\begin{tabular}{|c|c|}
\hline $\begin{array}{l}\text { Nevertheless, studies continue to be published } \\
\text { regularly that treat partner violence as a } \\
\text { unitary phenomenon, many of them claiming } \\
\text { to provide further evidence on the gender } \\
\text { symmetry issue. For example, Archer's (2000) } \\
\text { influential meta-analysis of the evidence } \\
\text { regarding gender symmetry, in spite of citing } \\
\text { my } 1995 \text { article, essentially ignored the } \\
\text { proposed distinctions among types of violence } \\
\text { and concluded that women are slightly more } \\
\text { violent than men in heterosexual partnerships. }\end{array}$ & $\begin{array}{l}\text { Pourtant, des études traitant de la violence par } \\
\text { partenaire comme d'un phénomène uniforme sont } \\
\text { régulièrement publiées. Beaucoup d'entre elles } \\
\text { prétendent apporter de nouvelles preuves à la question } \\
\text { de la symétrie de genre dans les violences. Par exemple, } \\
\text { bien qu'Archer cite mon article de } 1995 \text { dans sa méta- } \\
\text { analyse influente (2000) sur la preuve de la symétrie de } \\
\text { genre dans les violences, il ignore dans l'ensemble les } \\
\text { distinctions proposées entre les types de violence et } \\
\text { conclut que les femmes sont légèrement plus violentes } \\
\text { que les hommes dans les relations hétérosexuelles. }\end{array}$ \\
\hline
\end{tabular}

\section{Réceptions ambiguës sur la position pro-symétrie}

71 Jasinski, Blumenstein et Morgan (2014) ou Nybergh, Enander, Krantz (2016) ont quant à elles utilisé la typologie de Johnson afin d'en vérifier la solidité et de vérifier si elle était applicable à des échantillons d'hommes ayant subi des violences. Bien qu'elles semblent en faveur d'une vision bidirectionnelle des VPI, ces deux études soulignent que la typologie de Johnson fut essentielle à la prise de conscience du fait que le degré de contrôle était primordial pour comprendre les différentes VPI. Elles utilisent et critiquent la typologie car elle leur semble insuffisante en l'état pour décrire ou comprendre les violences subies par des hommes. D'une part, elles trouvent peu, voire pas de violences qu'on pourrait, selon la typologie de Johnson, appeler terrorisme intime, dans les cas d'hommes battus étudiés, car l'usage de la force et de la contrainte sexuelle n'est que rarement ou difficilement mis en œuvre par les femmes qui exercent un contrôle psychologique ou émotionnel sur leur compagnon. D'autre part, elles trouvent que les définitions des quatre catégories de la typologie manquent de précisions. Par exemple, elles ne voient pas en quoi la violence circonstancielle (qui peut inclure un compagnon violent et une compagne non contrôlante) diffère de la violence de terrorisme intime, puisque battre physiquement est déjà un moyen de contrôler son partenaire. Enfin, dans les cas qu'elles étudient, elles trouvent que les violences contrôlantes, proches du terrorisme intime, ont des conséquences très différentes sur les hommes qui ne semblent pas subir le même impact en termes de PTSD, d'incapacité de travail, etc. que les femmes.

72 Ces deux enquêtes appellent à de nouvelles typologies ou nouveaux critères pour construire les questionnaires sur les VPI, afin de mesurer de façon plus précise le degré de contrôle accompagnant les violences physiques exercées, notamment à travers des questions sur les contraintes sexuelles et les violences verbales. 


\section{Réception féministe : Lapierre \& Côté sur les risques de récupération antiféministes}

Deux chercheur'es féministes canadien'nes, Simon Lapierre et Isabelle Côté, explicitent dans deux contributions $(2014,2018)$ les risques de réutilisation décontextualisée de la typologie de Johnson par des masculinistes ou antiféministes.

Même si une étude bibliographique plus approfondie et systématique devrait être menée par les sociologues pour vérifier l'authenticité d'un tel risque, cette mise en garde rappelle les risques de simplifications et détournements de précédents travaux. En effet, étant donné que Johnson dit que les chiffres du terrorisme intime sont plus rares que ceux de la violence de couple circonstancielle et que les échantillons des féministes (comme ceux des spécialistes des violences familiales) ne sont pas représentatifs des VPI de toute la société, un détournement semble inévitable. Or, un mésusage de la typologie peut mener à « un renforcement du discours sur la symétrie de la violence» (p. 76), c'est-à-dire à " une vision de la violence faite aux femmes en contexte conjugal comme étant un phénomène rare et isolé dans notre société » ou à «la perception du terrorisme intime comme étant le fait d'une minorité de situations prises en charge par les services", avec des "répercussions sur la formulation des politiques gouvernementales en matière de violence conjugale » (p. 75).

Il me semble cependant essentiel de prolonger son travail, notamment autour de la création d'outils théoriques et pratiques pour mesurer le degré de contrôle exercé dans les VPI. Cela semble la manière la plus efficace de lutter contre les conséquences désastreuses du contrôle des femmes en tant que groupe social, et de contrer méthodologiquement les réutilisations malhonnêtes des catégories et autres outils élaborés par les spécialistes des violences par partenaire intime. A cette fin, toute étude de la violence par partenaire (théorie, typologie, enquête) devrait prendre en compte le contexte dans lequel la violence est exercée au niveau du couple, mais aussi au niveau de la société.

\section{Méthodologie et objectifs des enquêtes sur les VPI : sortir des typologie pour enquêter sur les institutions}

Pour aller plus loin, il est intéressant, comme a pu le faire Pauline Delage dans son étude comparative des acteurices et organismes de la prise en charge des VPI en France et aux États-Unis (2017) de s'éloigner des typologies de violence fondées sur les bourreaux ou les victimes pour s'intéresser aux institutions publiques. Dans sa thèse écrite en 1997, Safety for Battered Women In a textually Mediated Legal System, Ellen Pence critique les typologies et catégories de violence conjugale simplistes du bourreau et de la victime et observe la prise en charge des VPI par les institutions policières, sociales et judiciaires qu'elle juge défectueuse (désorganisation, inefficacité, discriminations). Elle remarque que les procédures et routines policières et judiciaires sont influencées idéologiquement et prennent en charge les affaires de violence conjugale comme des cas ponctuels de violences isolées au lieu de les étudier comme des cas de maltraitance conjugale dont l'évolution est progressive et itérative (p. 6). Ainsi, sur le terrain observé par Pence, chaque cas de violence conjugale à Duluth passe en moyenne par «six niveaux de gouvernement différents, une douzaine d'agences, et une trentaine 
d'individus » avec des formulaires et interrogatoires à chaque étape (p. 3). Pence estime que ces institutions ne sont jamais évaluées en retour par les victimes, alors que leur dysfonctionnement cause persistance et intensification des violences subies après le dépôt de plainte. Le mauvais accueil du dépôt de plainte (misogynie, racisme, formalités procédurières) et la lenteur bureaucratique, peuvent en effet mener à des rétractations et abandons de charges :

La plupart des recherches actuelles en sciences sociales [...] traduisent l'expérience vécue des femmes en catégories et en typologies qui nient leurs expériences réelles et les transportent dans le même monde discursif que celui que le système juridique utilise [...]. On identifie les femmes en termes institutionnels. Par exemple, une femme peut être une «témoin qui se rétracte». Cette catégorie ne dit rien de son expérience. [...] Je cite[rai] un extrait d'une entrevue avec une femme accusée d'avoir déposé un faux rapport de police quand elle vient retirer sa déclaration originale à la police. La rétractation ne dit rien des menaces, du rôle de l'avocat de la défense dans l'obtention de sa déclaration de rétractation, ou du délai de 11 mois entre l'agression et l'action du tribunal dans l'affaire. Elle occulte les relations sociales qui façonnent sa vie quotidienne, mais le/la sociologue utilise comme des «faits» les interprétations de l'institution juridique. Le tribunal, le jury et le sociologue n'ont pas accès à ce qui a réellement causé la déclaration de rétractation. L'incapacité du sociologue à échapper aux pratiques conceptuelles de l'appareil gouvernemental a laissé le psychologue nous parler des femmes qui sont battues, le criminologue des hommes qui battent et le théoricien juridique de l'efficacité des différentes approches juridiques et du travail avec différents types de criminels et victimes. Le regard est un regard à sens unique. La femme, objet de ce regard, est une source d'informations mais jamais de connaissance. Elle n'a jamais le droit de retourner le regard. C'est une créature qui n’a pas le droit de parler. (Pence 1997, p. $10)^{29}$

\section{Conclusion}

Après un bref rappel de l'enjeu sociétal de la transposition en France des débats étatsuniens sur la bidirectionnalité des violences (1), je me suis d'abord concentrée sur les aspects linguistiques et conceptuels posés par la traduction des catégories de violence de mon corpus. La catégorisation usuelle des violences a donné lieu à l'analyse morphosémantique de plusieurs matrices nominales qui questionnent non seulement le non-alignement des genres grammaticaux en français et en anglais mais aussi la difficulté de traduire l'ambiguïté directionnelle des violences inscrites des matrices lexicogéniques génériques anglaises par des néologismes avec des prépositions directionnelles en français (2). La catégorisation des violences produite par Johnson et les facteurs de violence étudiés par Renzetti ont donné lieu à une analyse sémantique et conceptuelle qui m'a donné l'occasion de questionner les typologies de violence centrées autour de la figure de la victime ou de l'agresseur, pour proposer une typologie complémentaire qui rappelle leur groupe social, et la façon dont les discriminations subies par ce groupe renforcent la violence par partenaire. J'ai ainsi proposé que des violences qui n'ont individuellement pas de visée patriarcale conscientisée peuvent produire un renforcement du contrôle social des femmes au profit d'un système social hétéronormatif encore largement patriarcal (3). J'ai par ailleurs proposé une analyse onomastique des bibliographies scientifiques au regard du genre pour rappeler l'importance de la présence du prénom en bibliographie pour améliorer la visibilité à long terme de la contribution des femmes à la recherche scientifique sur les VPI (4). Enfin, j'ai exposé les problèmes méthodologiques posés par 
la réutilisation des catégories de violences produites dans des études qui relèvent d'autres disciplines, d'autres sensibilités idéologiques, pour appeler à un renforcement théorique et méthodologique des études sur les violences par partenaire intime qui prennent en compte le contexte social de leur exercice et leur prise en charge par les institutions (5).

\section{BIBLIOGRAPHIE}

ADAMCZEWSKI, Henri \& DELMAS, Claude. 1998. Grammaire linguistique de l'anglais. 3e édition.

Paris : Armand Colin. 360 p.

ARCHER, John. 2000. « Sex differences in aggression between heterosexual partners: A metaanalytic review ", Psychological Bulletin vol. 126, $n^{\circ} 5$. pp. 651-680. Consulté le 3 mars 2020. URL : doi:10.1037/0033-2909.126.5.651.

BARD, Christine, DUPUIS-DÉRI, Francis \& BLAIS, Mélissa (dir.). 2019. Antiféminismes et masculinismes d'hier et d'aujourd'hui. Paris : Presses Universitaires de France. 512 p.

BEAUBATIE, Emmanuel. 2019. « «Le genre précède le changement de sexe. Parcours d'hommes et de femmes trans' en France ». », journée d'études « Matérialismes trans' » à l'ENS de Lyon, site Descartes.

BLONDIN, Odrée, OUELLET, Frédéric \& LECLERC, Chloé. 2018 « Les variations temporelles de la fréquence des violences physiques en contexte conjugal », Criminologie vol. 51, $\mathrm{n}^{\circ}$ 2. pp. 343-373. doi:10.7202/1051235ar.

BOUSCAREN, Janine \& CHUQUET, Jean. 2002. Grammaire et textes anglais. Paris : Editions Ophrys. $201 \mathrm{p}$.

CARDI, Coline \& PRUVOST, Geneviève. 2012. Penser la violence des femmes. Paris : La Découverte. Sciences Humaines. 448 p. Consulté le 15 avril 2019. URL : https://www.cairn.info/penser-laviolence-des-femmes--9782707172969.htm.

CAVALIN, Catherine. 2013. «Interroger les femmes et les hommes au sujet des violences conjugales en France et aux États-Unis : entre mesures statistiques et interprétations sociologiques ", Nouvelles Questions Feministes vol. 32, nº 1. pp. 64-76.

CHAMBERLAIN, Lori. 1988. « Gender and the Metaphorics of Translation », Signs. vol. 13, n 3. pp. 454-472.

CHANAY, Hugues Constantin de, CHEVALIER, Yannick \& GARDELLE, Laure. 2017. « Écrire le genre ", Mots. Les Langages du Politique $\mathrm{n}^{\circ} 113$.

CHEVALIER, Yannick. 2019. " "Etudiant·es" ou "Etudiant·e·s" : implications théoriques du pluriel dans les protocoles » in Genre et émancipation. Ile Congrès international de l'Institut du Genre. Angers. Consulté le 10 novembre 2020. URL : https://congresgenre19.sciencesconf.org/276630.

CULIOLI, Antoine. 1991. Pour une linguistique de l'énonciation: Opérations et représentations, tome 1. Gap : Ophrys. 225 p. 
DELAGE, Pauline. 2017. Violences conjugales : du combat féministe à la cause publique. Paris : Sciences Po-Les Presses. 262 p.

DERRIDA, Jacques. 1993. L'Oreille de l'autre. Montréal : Editions de l'Homme. 214 p.

DUCHESNE, Sophie. 2017. « Introduction. De l'analyse secondaire à la réanalyse. Une innovation méthodologique en débats ", Recherches Qualitatives. Juin, Hors-série, $\mathrm{n}^{\circ} 21$. ARQ Association pour la Recherche Qualitative. La réanalyse à l'épreuve de l'expérimentation. Textes en hommage à Annie-Claude Salomon. pp. 7-28.

DUMONT, Annie. 2017. « Les points de vue de la personnes exposées à la violence conjugale : complexité et importance de ce domaine d'étude ", Canadian Social Work Review / Revue canadienne de service social vol. 34, $\mathrm{n}^{\circ}$ 1. pp. 141-155. Consulté le 3 avril 2020. URL : doi:https://doi.org/ 10.7202/1040999ar.

EVANS, Jonathan \& FERNANDEZ, Fruela (dir.). 2018. The Routledge Handbook of Translation and Politics. London; New York : Routledge. 524 p.

FLOTOW, Luise von et KAMAL, Hala (dir.). 2020. The Routledge Handbook of Translation, Feminism and Gender. New York : Routledge. 574 p.

FRANCKEL, Jean-Jacques \& PAILLARD, Denis. 1998. « Aspects de la théorie d'Antoine Culioli », Langages vol. 32, $\mathrm{n}^{\circ} 129$, pp. 52-63. Consulté le 9 juin 2020. URL doi:10.3406/lgge.1998.2144.

GUILLAUME, Astrid. 2016. Traduction et implicites idéologiques. Besançon : La Völva. 208 p.

GUILLAUME, Astrid. 2016. Idéologie et traductologie. Paris : Editions L'Harmattan. 240 p.

GUILLAUMIN, Colette. 2016. Sexe, race et pratique du pouvoir. 1 édition. Paris : Les Éditions iXe. $234 \mathrm{p}$.

HANMER, Jalna. 1977. «Violence et contrôle social des femmes », Questions Féministes nº 1. pp. 68-88.

HÉRITIER, Françoise. 2012. Masculin/féminin I et II : La pensée de la différence. Odile Jacob. 326 p.

JASINSKI, Jana, BLUMENSTEIN, Lindsey et MORGAN, Rachel. 2014. « Testing Johnson's Typology : Is There Gender Symmetry in Intimate Terrorism? », Violence \& Victims. Février, vol. $29, \mathrm{n}^{\circ} 1$. Springer Publishing Company, Inc. pp. 73-88. Consulté le 19 septembre 2019, URL : doi: 10.1891/0886-6708.VV-D-12-00146.

JOHNSON, Michael. 2006. «Conflict and Control: Gender Symmetry and Asymmetry in Domestic Violence ", Violence against women vol. 12. pp. 1003-1018. Consulté le 9 juin 2020. URL : doi: $10.1177 / 1077801206293328$.

KRIPKE, Saul. 1982. La logique des noms propres. Paris : Éditions de Minuit. 173 p.

LAPIERRE, Simon \& CÔTÉ, Isabelle. 2014. « La typologie de la violence conjugale de Johnson : quand une contribution proféministe risque d'être récupérée par le discours masculiniste et antiféministe ", Intervention $\mathrm{n}^{\circ} 140$. pp. 69-79.

LAPIERRE, Simon, CÔTÉ, Isabelle, BUETTI, David, LAMBERT, Amélie, LESSARD, Geneviève \& DROLET, Marie. 2017. « Conflits entre conjoints ou contrôle des hommes sur les femmes? L'expérience et le point de vue d'enfants et d'adolescents exposés à la violence conjugale ", Enfances Familles Générations. Revue interdisciplinaire sur la famille contemporaine $\mathrm{n}^{\circ} 22$. Consulté le 13 octobre 2017, URL : https://efg.revues.org/461.

LEEDER, Elaine. 1988. « Enmeshed in Pain », Women \& Therapy vol. 7, nº 1. pp. 81-99. Consulté le 14 juin 2020. URL : doi:10.1300/J015V07N01_07. 
LENCLUD, Gérard, CLAVERIE, Elisabeth \& JAMIN, Jean. 1984. « Présentation : Une ethnographie de la violence est-elle possible? ", Études rurales vol. 95, n 1. pp. 9-21.

LESSARD, Geneviève, MONTMINY, Lyse, LESIEUX, Élisabeth, FLYNN, Catherine, ROY, Valérie, GAUTHIER, Sonia \& FORTIN, Andrée. 2015. « Les violences conjugales, familiales et structurelles : vers une perspective intégrative des savoirs », Enfances Familles Générations. Revue interdisciplinaire sur la famille contemporaine $\mathrm{n}^{\circ} 22$. Consulté le 20 septembre 2017, URL : http:// efg.revues.org.acces.bibliotheque-diderot.fr/425.

MARIGNIER, Noémie. 2015. «L'agentivité en question : étude des pratiques discursives des femmes enceintes sur les forums de discussion ", Langage et société $\mathrm{n}^{\circ} 152, \mathrm{n}^{\circ}$ 2. pp. 41-56.

NAEPELS, Michel. 2006. «Quatre questions sur la violence », L'Homme. Revue française d'anthropologie $\mathrm{n}^{\circ}$ 177-178. pp. 487-495. Consulté le 20 septembre 2017, URL : doi:10.4000/lhomme. 21787.

NYBERGH, Lotta, ENANDER, Viveka et KRANTZ, Gunilla. 2016. « Theoretical Considerations on Men's Experiences of Intimate Partner Violence: An Interview-Based Study », Journal of Family Violence vol. 31, $\mathrm{n}^{\circ}$ 2. pp. 191-202. Consulté le 19 septembre 2018, URL : doi:10.1007/ s10896-015-9785-8.

PENCE, Ellen. 1996. Safety for battered women in a textually mediated legal system. Toronto : Department of Sociology in Education University of Toronto.

PENCE, Ellen \& PAYMAR, Michael. 1993. Education Groups for Men Who Batter: The Duluth Model. New YorkSpringer Publishing Co Inc. $212 \mathrm{p}$.

PETIT, Michel. 1991. « Le génitif en 's, le génitif en of et la structure $\mathrm{N} \varnothing \mathrm{N}$ avec les noms propres dans le discours mathématique en anglais ", Cahiers de l'APLIUT vol. 10, n ${ }^{\circ}$. pp. 83-99. Consulté le 16 juin 2017, URL : doi:10.3406/apliu.1991.2192.

PLAG, Ingo. 2003. Word-Formation in English. Cambridge : Cambridge University Press. 256 p.

RENZETTI, Claire M. 1992. Violent Betrayal : Partner Abuse in Lesbian Relationships. 1 édition. SAGE Publications, Inc. 208 p.

RENZETTI, Claire M \& LEE, Raymond M (dir.). 1993. Researching Sensitive Topics. Thousand Oaks, CASAGE Publications, Inc. $312 \mathrm{p}$.

REY, Alain. 2019. Le Dictionnaire Historique de la langue française - Coffret compact 3 volumes - Nouvelle édition augmentée. Le Robert. 4416 p.

ROGERS, Rebecca \& MOLINIER, Pascale. 2016. Les femmes dans le monde académique : Perspectives comparatives. PUR. 226 p.

SABLAYROLLES, Jean-François. 1997. « Néologismes : une typologie des typologies », Cahier du CIEL 1996-1997. p. 11-48.

SAÏDI, Samantha. 2018. Traduire Michael P. Johnson et Claire Renzetti [Mémoire de master]. Lyon : Université de Lumière Lyon 2. 292 p.

SAÏDI, Samantha \& MOZZICONACCI, Vanina. 2018. « Projet FELiCiTE : Féminismes en Ligne, Circulations, Traductions, Editions ", in Journée d'étude Traduction et outils numériques, 5 avril 2018, Lyon, ENS de Lyon. Consulté le 10 novembre 2020. URL : http://triangle.ens-lyon.fr/spip.php? article7623.

SAIIDI, Samantha, MOZZICONACCI, Vanina, THOMAS, Héloïse \& ORAZI, Françoise. 2018.

«FELiCiTE : Féminismes en Ligne, Circulations, Traductions, Editions », in Journée d'études de lancement du séminaire de traductologie féministe FELiCiTE. ENS de Lyon. 
SAÏDI, Samantha. 2001. "Why don't you go? An utterer-centered approach of Interrogation-negations in Interviews » [Mémoire de master]. Nantes. Université de Nantes. 89 p.

SELESKOVITCH, D. \& LEDERER, M. 2014. Interpréter pour traduire. Nouvelle édition revue et Corrigée. Paris : Les Belles Lettres. 480 p.

SIBLOT, Paul. 1997. « Nomination et production de sens : le praxème », Langages vol. 31, $\mathrm{n}^{\circ} 127$. pp. 38-55. doi :10.3406/lgge.1997.2124.

SMALL, Mario Luis. 2009. « "How many cases do I need?": On science and the logic of case selection in field-based research », Ethnography vol. 10, $\mathrm{n}^{\circ}$ 1. pp. 5-38. doi:

$10.1177 / 1466138108099586$.

STARK, Evan. 2012. « Re-presenting Battered Women: Coercive Control and the Defense of Liberty ", in Violence Against Women : Complex Realities and New Issues in a Changing World. Presses de l'Université du Québec. Québec. p. 19. Consulté le 10 novembre 2020. URL : https://

www.welshwomensaid.org.uk/wp-content/uploads/2017/08/

evan_stark_article_final_100812.pdf.

STARK, Evan. 2007. Coercive Control: How Men Entrap Women in Personal Life (Interpersonal Violence). Oxford : Oxford University Press. 464 p. Consulté le 15 avril 2019, URL : https://www.amazon.fr/ Coercive-Control-Entrap-Women-Personal-ebook/dp/B00VQVNCES/ref=sr_1_6?

keywords=Evan+stark\&link_code=qs\&qid=1555347613\&s=gateway\&sourceid=Mozillasearch\&sr=8-6.

STARK, Evan. 2006. « Commentary on Johnson's “Conflict and Control: Gender Symmetry and Asymmetry in Domestic Violence" ", Violence Against Women. vol. 12, n 11. pp. 1019-1025. doi: 10.1177/1077801206293329.

TOURNIER, Jean. 2007. Introduction descriptive à la lexicogénétique de l'anglais contemporain. Genève : Editions Slatkine. $528 \mathrm{p}$.

WALKER, Lenore E. 1989. Terrifying love: why battered women kill and how society responds. New York : N.Y.Harper Perennial. $352 \mathrm{p}$.

WITTIG, Monique. 1978. La Pensée straight. 1 édition. Paris : Amsterdam. 200 p.

YANG-PAYA, My-Kim \& MARCOVICI, Céline. 2016. Guide juridique des femmes victimes de violences. Paris : Alma éditeur. 128 p.

\section{NOTES}

1. À noter également le Grenelle des violences conjugales dont les résultats ont été annoncés en octobre 2019.

2. Mémoire de traduction de master sous la direction d'Anne Verjus, Pascal Bataillard et Niall Bond. Il s'agit entre autres des textes de Michael P. Johnson «Conflict and Control: Gender, symmetry, and asymmetry in domestic violence ». Violence Against Women 12, 2006 : 1003-1018, et de Claire Renzetti, Violent Betrayal: Partner Abuse in Lesbian Relationships, $1^{\mathrm{e}}$ éd., SAGE Publications, 1992. Ce projet n'aurait pas vu le jour sans Annie Lechenet, Vanina Mozziconacci et Pauline Delage.

3. Sur le projet, voir http://reanalyse.hypotheses.org/projet-anr.

4. Pour une justification de la traduction de intimate partner violence par violence par partenaire intime, lire la partie 2 . 
5. La traductologie féministe est la traduction que nous avons donnée en 2017 d'une sousdiscipline peu reconnue en France, celle des Feminist Translation Studies qui étudie, entre autres, comme le préconisait Lori Chamberlain dès 1988 (cf. traduction dans ce numéro): la reconnaissance des femmes autrices et traductrices, les pratiques ou métaphores de la traduction, le genre linguistique dans les traductions et la circulation des idées en études de genre.

6. Cette relation dichotomique est analysée avec la Théorie des opérations énonciatives en partie 1 : «identification» et «rupture» sont des possibilités du parcours de la personne (« identification » et « rupture » et « différentiation »).

7. Selon le souhait de l'autrice, cet article n'applique pas les règles orthographiques de 1990 en vigueur dans les autres textes de la revue GLAD!.

8. La cognition se dit de tous les processus mentaux (ou processus cognitifs) liés à la connaissance comme l'acquisition, la mémoire, le langage, le raisonnement, l'attention, etc. Pour Antoine Culioli, dont l'approche constructiviste se distingue pourtant de la linguistique cognitive - née vers 1956 et développée en parallèle aux États-Unis - la cognition est une notion difficile à définir, et «dangereusement ambiguë » $(1995$, p. 31) : pour lui, la cognition comprend toujours l'affect (1990: p. 21) et peut être représentée au premier niveau des trois niveaux de représentations qu'il dégage de l'énonciation (celui des représentations mentales ou cognitives, celui des représentations linguistiques ou textuelles et celui des représentations métalinguistiques) (Fuchs, 2008). Ici, nous tenterons d'expliciter certaines catégories sur ces trois niveaux: mentaux (représentations individuelles même si elles se font en interaction) et textuels (dans notre contexte d'études, les catégories disciplinaires) grâce au niveau de représentation métalinguistiques. L'objectif n'est pas ici de comprendre ce qui se joue dans les VPI d'un point de vue cognitif, ce qui pourrait être l'objet d'autres études.

9. La roue de la violence et du pouvoir, utilisée pour la première fois par le projet Duluth, montrait les différents aspects du pouvoir et du contrôle qui précèdent et aggravent la violence (isolement, agression économique, intimidation, menace, machisme, dévalorisation, agression sexuelle, chantage aux enfants, etc.). Elle est aujourd'hui controversée pour son aspect cyclique qui omet de représenter l'agentivité des victimes et la sortie de la violence: http:// www.ncdsv.org/images/PowerControlwheelNOSHADING.pdf (consulté le 26 novembre 2020).

10. Rapport du Ministère de l'Intérieur «Viols, tentatives de viol et attouchements sexuels Interstats Analyse $\mathrm{N}^{\circ} 18$ - Décembre $2017 »$ : https://www.interieur.gouv.fr/Interstats/Actualites/ Viols-tentatives-de-viol-et-attouchements-sexuels-Interstats-Analyse-N-18-Decembre-2017 (consulté le 26 novembre 2020).

11. Rapport du Ministère de la Justice "Les condamnations pour violences sexuelles» du Ministère de la justice - Chiffre Infostat Justice, le 6 septembre 2018 : http://www.justice.gouv.fr/ art_pix/stat_Infostat_164.pdf trouvé sur cette page: http://www.justice.gouv.fr/ statistiques-10054/infostats-justice-10057/les-condamnations-pour-violencessexuelles-31757.html (consulté le 26 novembre 2020).

12. Rapport «Les victimes du sexisme en France du Ministère de la justice Chiffre Interstats Analyse $n^{\circ} 19$.- Mars $2019 »$ : https://www.interieur.gouv.fr/Interstats/Actualites/Insecurite-etdelinquance-en-2017-premier-bilan-statistique

(consulté le 26 novembre 2020). Dans le Tableau «Crimes et délits à caractère sexiste enregistrés en 2017 par les forces de sécurité (nombre de victimes, répartition par infraction, et part des femmes)", on trouve les groupes suivants : deuxième groupe infractionnel : crimes et délits commis au sein du couple: Viol par conjoint (y compris tentatives) 2410 dont 2370 femmes (98\%). Troisième groupe infractionnel : crimes et délits à caractère sexuel (hors infractions dans le cadre conjugal) : Viol par conjoint (y compris tentatives) 14700, dont 12564 femmes (85\%). 
13. Rapport du Haut Conseil à l'Égalité entre les femmes et les hommes, Violences de Genre : Repères statistiques: http://www.haut-conseil-egalite.gouv.fr/violences-de-genre/reperes-statistiques/ (consulté le 29 octobre 2019).

14. Le Point, «Féminicides : 121 femmes tuées en 2018 par leur conjoint ou ex-compagnon », 10 juillet 2019: https://www.lepoint.fr/societe/feminicides-121-femmes-tuees-en-2018-par-leurconjoint-ou-ex-compagnon-10-07-2019-2323920_23.php (consulté le 4 novembre 2019).

15. Rapport du Ministère de l'Intérieur Délégation aux Victimes, Étude nationale sur les morts violentes au sein du couple en 2018.

16. Recensement des féminicides conjugaux en France Collectif, « 2019 - Féminicides par (ex) compagnon », Google My Maps, Consulté le 5 mai 2020, URL : https://www.google.com/maps/d/ viewer?mid=1Y9bFj8Cjfl3rKwuyDBB5-LNkdKKAjtq9 et article de France Inter: https:// www.franceinter.fr/societe/2019-l-annee-ou-le-feminicide-s-est-impose-dans-la-societefrancaise.

17. " Trois personnes transgenres sauvagement agressées à Paris par une "brigade anti-trav" " : http://www.leparisien.fr/paris-75/paris-trois-transgenres-sauvagement-agresses-par-une-

brigade-anti-trav-29-03-2018-7636569.php (consulté le 6 mai 2020).

18. La justification de l'usage de la Théorie des Opérations énonciatives d'Antoine Culioli est explicitée dans la partie 1.

19. Ma traduction ici revue à partir d'un précédent travail (Saïdi 2018).

20. Pour une analyse de cette catégorie intimate partner violence, lire partie 2.

21. Ma traduction ici revue à partir d'un précédent travail (2018).

22. Christine Delphy (2013) définit ainsi le patriarcat: «1) le patriarcat est le système de subordination des femmes aux hommes dans les sociétés industrielles contemporaines 2) ce système a une base économique 3) cette base est le mode de production domestique ». Elle en tire une théorie du patrimoine comme un mode de circulation des biens et donc comme un lieu économique qui : «1) n'est pas caractérisé par l'échange, mais par le don 2) les acteurs ne sont pas interchangeables mais définis très étroitement par les règles de la parenté 3) enfin cette circulation ne dépend pas du bon vouloir des acteurs : ni des donateurs, ni des bénéficiaires. »

23. Despentes Virginie, "Césars : "Désormais on se lève et on se barre", par Virginie Despentes ", Libération.fr, 1 mars 2020, Consulté le 6 mai 2020, URL: https://www.liberation.fr/debats/ 2020/03/01/cesars-desormais-on-se-leve-et-on-se-barre_1780212.

24. Témoignage paru en novembre 2019: https://www.mediapart.fr/journal/france/031119/ metoo-dans-le-cinema-l-actrice-adele-haenel-brise-un-nouveau-tabou

25. Voir cette vidéo de Charlie Brousseau qui définit l'hétéronorme ainsi : "l'hétéronorme c'est l'injonction à être en couple dans une relation de genre binaire [...] avec relation de domination » https://www.youtube.com/watch?v=ZstZ8fIHngs (consulté le 20 mars 2020).

26. Pour l'aspect méthodologique je n'étudie ici que la réception de Johnson: la même chose pourrait être effectuée pour le travail de Renzetti.

27. Ma traduction ici revue à partir d'un précédent travail (2018).

28. Ma traduction ici revue à partir d'un précédent travail (2018).

29. Ma traduction ici. 


\section{RÉSUMÉS}

Cet article propose une traductologie politique qui s'attarde sur la circulation des typologies de violences adoptées par le champ d'étude pluridisciplinaire des violences par partenaires intimes (VPI), et sur les méthodes de cette circulation (traductions, ré-analyses, ...). Interrogeant les préjugés de genre véhiculés dans les traductions ou réutilisations francophones de ces catégorisations génériques produites par deux sociologues féministes états-uniennes, Michael Johnson (2006) et Claire Renzetti (1992), l'article en propose une explicitation linguistique et sémantique. Cette étude confirme que leurs traductions ou réutilisations charrient des traces d'impensés ou préjugés de genre, souvent imputables à ceux et celles qui traduisent, si ce n'est aux auteur·ice-s. Ainsi les termes de violence lesbienne, du partenaire intime ou symétrie des violences, mobilisés dans les traductions ou réutilisations de ces textes, ne sont pas neutres mais véhiculent l'idée d'une essentialisation masculine ou lesbienne de la violence et d'une symétrie genrée des violences, ce qui est justement remis en question par les grilles d'analyse de Johnson et Renzetti.

This article proposes a social and political translation study that focuses on the circulation and methods of circulation (translation, re-use, re-analysis, ...) of the categories of violence adopted by the multidisciplinary field studying intimate partner violence (IPV). Questioning gender prejudices conveyed in French translations or re-uses of categorizations produced by two American feminist sociologists, Johnson (2006) and Renzetti (1992), the article proposes a linguistic and semantic explanation. This study confirms that their translations or reuses carry traces of gender prejudices that we can often attribute to those who translate if not to the authors. Thus, the terms lesbian violence, intimate partner violence or symmetry of violence used in the translations or reuses of these texts are not neutral but convey the idea of a masculine or lesbian essentialization of violence, or of a vertical or horizontal complementarity between men and women in violence, which is denounced by the feminist perspectives used by Johnson and Renzetti.

\section{INDEX}

Thèmes : Recherches

Mots-clés : traduction, genre, linguistique, rapport de pouvoir, violences

Keywords : translation, gender, linguistics, power relations, violences

\section{AUTEUR}

\section{SAMANTHA SAÏDI}

ENS de Lyon

Samantha Saìdi est ingénieure d'études en humanités numériques et traductrice anglais-français en sciences humaines et sociale à l'ENS de Lyon, rattachée au laboratoire Triangle. Après des études de linguistique et en sciences de l'information, elle a travaillé à l'édition numérique de différents corpus de sciences humaines et sociales comme L'Écho de la Fabrique, La Storia d'Italia, La bibliothèque Foucaldienne ou Musea. Elle est co-fondatrice du projet FELiCiTE (Féminismes en Ligne : Circulations, Traductions, Éditions). 